\title{
Higgcision in the two-Higgs doublet models
}

\author{
Kingman Cheung, ${ }^{a, b}$ Jae Sik Lee ${ }^{c}$ and Po-Yan Tseng ${ }^{a}$ \\ ${ }^{a}$ Department of Physics, National Tsing Hua University, \\ Hsinchu 300, Taiwan \\ ${ }^{b}$ Division of Quantum Phases and Devices, School of Physics, Konkuk University, \\ Seoul 143-701, Republic of Korea \\ ${ }^{c}$ Department of Physics, Chonnam National University, \\ 300 Yongbong-dong, Buk-gu, Gwangju, 500-75\%, Republic of Korea \\ E-mail: cheung@phys.nthu.edu.tw, jslee@jnu.ac.kr, \\ d9722809@oz.nthu.edu.tw
}

ABSTRACT: We perform global fits to general two-Higgs doublet models (2HDMs) with generalized couplings using the most updated data from ATLAS, CMS, and Tevatron. We include both scenarios with CP-conserving and CP-violating couplings. By relaxing the requirement on the discrete symmetries that are often imposed on the Yukawa couplings, we try to see which of the $2 \mathrm{HDM}$ is preferred. We found that (i) Higgcision in $2 \mathrm{HDMs}$ can be performed efficiently by using only 4 parameters including the charged Higgs contributions to the Higgs couplings to two photons, (ii) the differences among various types of $2 \mathrm{HDMs}$ are very small with respect to the chi-square fits, (iii) $\tan \beta$ is constrained to be small, (iv) the $p$-values for various fits in $2 \mathrm{HDM}$ are worse than that of the standard model. Finally, we put emphasis on our findings that future precision measurements of the Higgs coupling to the scalar top-quark bilinear $\left(C_{u}^{S}\right)$ and $\tan \beta$ may endow us with the discriminating power among various types of $2 \mathrm{HDMs}$ especially when $C_{u}^{S}$ deviates from its SM value 1 .

Keywords: Phenomenological Models

ARXIV EPRINT: 1310.3937 


\section{Contents}

1 Introduction 1

2 Formalism 2

$\begin{array}{lll}3 & 2 H D M s & 3\end{array}$

4 Fits $\quad 8$

4.1 CP conserving fits 9

$\begin{array}{llr}4.1 .1 & \mathrm{CPC} 2 & 10\end{array}$

4.1.2 CPC3 13

$\begin{array}{lll}4.2 & \mathrm{CP} \text { violating fits } & 21\end{array}$

$\begin{array}{lll}4.2 .1 & \text { CPV3 } & 21\end{array}$

$\begin{array}{lll}4.2 .2 & \text { CPV4 } & 23\end{array}$

5 Discussion $\quad 34$

\section{Introduction}

Upon the observation of a new boson at a mass around $125 \mathrm{GeV}$ at the Large Hadron Collider (LHC) [1, 2], the Higgs precision (Higgcision) era has just begun. A study based on a generic framework for the deviations of the couplings from their standard model (SM) values shows [6] that the SM Higgs boson [3-5] provides the best fit to all the most updated Higgs data from ATLAS [7, 8], CMS [9-12], and Tevatron [13, 14].

In addition to a number of more or less model-independent studies [15-42], there are also studies done in the 2HDM [43-60] and supersymmetric [61-65] frameworks. In this work, we perform global fits to the general 2HDMs (Higgcision in 2HDMs) closely following the generic framework suggested in ref. [6]. We use the most updated data from the ATLAS, CMS, and the Tevatron and include the scenarios with CP-conserving (CPC) and $\mathrm{CP}$-violating (CPV) couplings. We find that Higgcision in 2HDMs can be performed very efficiently by using only 3 parameters $\left(C_{u}^{S}, C_{u}^{P}\right.$, and $\tan \beta$, as shown later), if one can neglect the charged-Higgs contribution to the Higgs couplings to two photons. To consider the case when the charged-Higgs contribution to the $H \gamma \gamma$ couplings is significant, one may need only one additional parameter.

Furthermore, we relax the requirement on the discrete symmetries, which are often imposed on the Yukawa couplings to guarantees the absence of tree-level Flavor Changing Neutral Current (FCNC) [66], to see which of the 2HDMs is preferred. We find that the differences in the chi-squares among various types of $2 \mathrm{HDM}$ are very small and one cannot see any preferences in both the CP-conserving and CP-violating cases. 
A number of important findings in this work are:

1. the SM provides the best fit in terms of $p$-values. The general $2 \mathrm{HDM}$ fits at most improve marginally in the total $\chi^{2}$ at the expense of additional parameters though, and so the $p$-values do not improve at all;

2. the differences among various types of $2 \mathrm{HDM}$ are negligible in fitting the Higgs data;

3. the gauge boson coupling $C_{v}$ is constrained to be close to 1 , which means that the observed Higgs boson is responsible for the most part of the electroweak symmetry breaking; and

4. the $\tan \beta$ is constrained to a small value.

Finally, we emphasize that future precision measurements of $C_{u}^{S}$ and $\tan \beta$ can provide us with the discriminating power among various types of $2 \mathrm{HDMs}$ especially when $C_{u}^{S}$ deviates from its SM value 1.

The organization of the paper is as follows. In the next section, we describe the interactions of the Higgs bosons, including deviations in the Yukawa couplings and deviations in the loop functions of $H \gamma \gamma, H g g$, and $H Z \gamma$ vertices, as well as the notation used in the analysis. In section 3, we fix the Higgs potential and Yukawa couplings of the general 2HDMs under consideration and describe how to perform Higgcision in 2HDMs. We articulate that only 4 fitting parameters are needed if we concentrate on the couplings of the candidate for the $125 \mathrm{GeV}$ Higgs boson. We present the results of various fits in section 5 and conclude in section 6 .

\section{Formalism}

For the Higgs couplings to the SM particles assuming the Higgs boson is a generic CP-mixed state without carrying any definite CP-parity, we follow the conventions and notations of CPsuperH [67-69] in which the Higgs couplings to fermions are given as

$$
\mathcal{L}_{H \bar{f} f}=-\sum_{f=u, d, l} \frac{g m_{f}}{2 M_{W}} H \bar{f}\left(g_{H \bar{f} f}^{S}+i g_{H \bar{f} f}^{P} \gamma_{5}\right) f
$$

where $f=u, d, l$ stands for the up- and down-type quarks and charged leptons, respectively, and those to the massive vector bosons are

$$
\mathcal{L}_{H V V}=g M_{W}\left(g_{H W W} W_{\mu}^{+} W^{-\mu}+g_{H Z Z} \frac{1}{2 c_{W}^{2}} Z_{\mu} Z^{\mu}\right) H .
$$

In the SM, $g_{H \bar{f} f}^{S}=1, g_{H \bar{f} f}^{P}=0$, and $g_{H W W}=g_{H Z Z} \equiv g_{H V V}=1$. For the loop-induced Higgs couplings to two photons, two gluons and $Z \gamma$, and their relevance to the couplings $g_{H \bar{f} f}^{S, P}$ and $g_{H V V}$, we refer to refs. [6, 67-69]. Without loss of generality, we use the following 
notation for the parameters in the fits:

$$
\begin{aligned}
& C_{u}^{S}=g_{H \bar{u} u}^{S}, \quad C_{d}^{S}=g_{H \bar{d} d}^{S}, \quad C_{\ell}^{S}=g_{H \overline{l l}}^{S} ; \quad C_{v}=g_{H V V} ; \\
& C_{u}^{P}=g_{H \bar{u} u}^{P}, \quad C_{d}^{P}=g_{H \bar{d} d}^{P}, \quad C_{\ell}^{P}=g_{H \bar{l} l}^{P} \text {; } \\
& \Delta S^{\gamma}, \Delta S^{g}, \Delta P^{\gamma}, \Delta P^{g} ; \\
& \Delta \Gamma_{\text {tot }} \text {, }
\end{aligned}
$$

where $\Delta S^{\gamma}$ and $\Delta P^{\gamma}$ denote additional loop contributions to the loop factor $S^{\gamma}$ and $P^{\gamma}$, respectively; and similarly for $\Delta S^{g}$ and $\Delta P^{g}$. The $\Delta \Gamma_{\text {tot }}$ represents an additional nonstandard decay width of the Higgs boson (e.g., decay into the lighter Higgses). Here we assume generation independence and also custodial symmetry between the $W$ and $Z$ bosons.

Our analysis is based on the theoretical signal strength which may be approximated as the product

$$
\widehat{\mu}(\mathcal{P}, \mathcal{D}) \simeq \widehat{\mu}(\mathcal{P}) \widehat{\mu}(\mathcal{D})
$$

where $\mathcal{P}=\mathrm{ggF}, \mathrm{VBF}, \mathrm{VH}, \mathrm{ttH}$ denote the production mechanisms and $\mathcal{D}=\gamma \gamma, Z Z, W W$, $b \bar{b}, \tau \bar{\tau}$ the decay channels. For explicit expressions of $\widehat{\mu}(\mathcal{P})$ and $\widehat{\mu}(\mathcal{D})$, we again refer to ref. [6], but by noting they are basically given by the ratios of the Higgs couplings to the corresponding SM ones.

\section{$3 \quad 2 \mathrm{HDMs}$}

The general 2HDM potential may be given by [70]

$$
\begin{aligned}
\mathrm{V}= & -\mu_{1}^{2}\left(\Phi_{1}^{\dagger} \Phi_{1}\right)-\mu_{2}^{2}\left(\Phi_{2}^{\dagger} \Phi_{2}\right)-m_{12}^{2}\left(\Phi_{1}^{\dagger} \Phi_{2}\right)-m_{12}^{* 2}\left(\Phi_{2}^{\dagger} \Phi_{1}\right) \\
& +\lambda_{1}\left(\Phi_{1}^{\dagger} \Phi_{1}\right)^{2}+\lambda_{2}\left(\Phi_{2}^{\dagger} \Phi_{2}\right)^{2}+\lambda_{3}\left(\Phi_{1}^{\dagger} \Phi_{1}\right)\left(\Phi_{2}^{\dagger} \Phi_{2}\right)+\lambda_{4}\left(\Phi_{1}^{\dagger} \Phi_{2}\right)\left(\Phi_{2}^{\dagger} \Phi_{1}\right) \\
& +\frac{\lambda_{5}}{2}\left(\Phi_{1}^{\dagger} \Phi_{2}\right)^{2}+\frac{\lambda_{5}^{*}}{2}\left(\Phi_{2}^{\dagger} \Phi_{1}\right)^{2}+\lambda_{6}\left(\Phi_{1}^{\dagger} \Phi_{1}\right)\left(\Phi_{1}^{\dagger} \Phi_{2}\right)+\lambda_{6}^{*}\left(\Phi_{1}^{\dagger} \Phi_{1}\right)\left(\Phi_{2}^{\dagger} \Phi_{1}\right) \\
& +\lambda_{7}\left(\Phi_{2}^{\dagger} \Phi_{2}\right)\left(\Phi_{1}^{\dagger} \Phi_{2}\right)+\lambda_{7}^{*}\left(\Phi_{2}^{\dagger} \Phi_{2}\right)\left(\Phi_{2}^{\dagger} \Phi_{1}\right) .
\end{aligned}
$$

With the parameterization

$$
\Phi_{1}=\left(\begin{array}{c}
\phi_{1}^{+} \\
\frac{1}{\sqrt{2}}\left(v_{1}+\phi_{1}^{0}+i a_{1}\right)
\end{array}\right) ; \quad \Phi_{2}=\mathrm{e}^{i \xi}\left(\begin{array}{c}
\phi_{2}^{+} \\
\frac{1}{\sqrt{2}}\left(v_{2}+\phi_{2}^{0}+i a_{2}\right)
\end{array}\right)
$$

and denoting $v_{1}=v \cos \beta=v c_{\beta}$ and $v_{2}=v \sin \beta=v s_{\beta}$, one may remove $\mu_{1}^{2}, \mu_{2}^{2}$, and $\Im \mathrm{m}\left(m_{12}^{2} \mathrm{e}^{i \xi}\right)$ from the $2 \mathrm{HDM}$ potential using three tadpole conditions. Then, including the vacuum expectation value $v$, one may need the following 13 parameters plus one sign:

$$
\begin{aligned}
& v, t_{\beta},\left|m_{12}\right| ; \\
& \lambda_{1}, \lambda_{2}, \lambda_{3}, \lambda_{4},\left|\lambda_{5}\right|,\left|\lambda_{6}\right|,\left|\lambda_{7}\right| ; \\
& \phi_{5}+2 \xi, \phi_{6}+\xi, \phi_{7}+\xi, \operatorname{sign}\left[\cos \left(\phi_{12}+\xi\right)\right],
\end{aligned}
$$

to fully specify the general $2 \mathrm{HDM}$ potential. Here $m_{12}^{2}=\left|m_{12}\right|^{2} e^{i \phi_{12}}$ and $\lambda_{5,6,7}=\left|\lambda_{5,6,7}\right|$ $e^{i \phi_{5,6,7}}$ and we note that $\sin \left(\phi_{12}+\xi\right)$ is fixed by the CP-odd tadpole condition when the 


\begin{tabular}{|c|cccc|}
\hline & 2HDM I & 2HDM II & 2HDM III & 2HDM IV \\
\hline$\eta_{1}^{d}$ & 0 & 1 & 0 & 1 \\
$\eta_{2}^{d}$ & 1 & 0 & 1 & 0 \\
\hline$\eta_{1}^{l}$ & 0 & 1 & 1 & 0 \\
$\eta_{2}^{l}$ & 1 & 0 & 0 & 1 \\
\hline
\end{tabular}

Table 1. Classification of 2HDMs satisfying the Glashow-Weinberg condition [66] which guarantees the absence of tree-level FCNC.

CP phases $\phi_{5}+2 \xi, \phi_{6}+\xi$ and $\phi_{7}+\xi$ are given and, accordingly, $\cos \left(\phi_{12}+\xi\right)$ is determined up to the two-fold ambiguity. One may take the convention with $\xi=0$ without loss of generality.

On the other hand, the Yukawa couplings are given in the interactions

$$
\begin{aligned}
-\mathcal{L}_{Y}= & h_{u} \overline{u_{R}} Q^{T}\left(i \tau_{2}\right) \Phi_{2}+h_{d} \overline{d_{R}} Q^{T}\left(i \tau_{2}\right)\left(-\eta_{1}^{d} \widetilde{\Phi}_{1}-\eta_{2}^{d} \widetilde{\Phi}_{2}\right) \\
& +h_{l} \overline{l_{R}} L^{T}\left(i \tau_{2}\right)\left(-\eta_{1}^{l} \widetilde{\Phi}_{1}-\eta_{2}^{l} \widetilde{\Phi}_{2}\right)+\text { h.c. }
\end{aligned}
$$

where $Q^{T}=\left(u_{L}, d_{L}\right), L^{T}=\left(\nu_{L}, l_{L}\right)$, and $\widetilde{\Phi}_{i}=i \tau_{2} \Phi_{i}^{*}$ with

$$
i \tau_{2}=\left(\begin{array}{cc}
0 & 1 \\
-1 & 0
\end{array}\right) .
$$

We note that there is a freedom to redefine the two linear combinations of $\Phi_{2}$ and $\Phi_{1}$ to eliminate the coupling of the up-type quarks to $\Phi_{1}[71]$. The $2 \mathrm{HDMs}$ are classified according to the values of $\eta_{1,2}^{l}$ and $\eta_{1,2}^{d}$ as in table 1 .

By identifying the couplings

$$
h_{u}=\frac{\sqrt{2} m_{u}}{v} \frac{1}{s_{\beta}} ; \quad h_{d}=\frac{\sqrt{2} m_{d}}{v} \frac{1}{\eta_{1}^{d} c_{\beta}+\eta_{2}^{d} s_{\beta}} ; \quad h_{l}=\frac{\sqrt{2} m_{l}}{v} \frac{1}{\eta_{1}^{l} c_{\beta}+\eta_{2}^{l} s_{\beta}},
$$

we have obtained the following Higgs-fermion-fermion interactions

$$
\begin{aligned}
-\mathcal{L}_{H_{i} \bar{f} f}= & \frac{m_{u}}{v}\left[\bar{u}\left(\frac{O_{\phi_{2} i}}{s_{\beta}}-i \frac{c_{\beta}}{s_{\beta}} O_{a i} \gamma_{5}\right) u\right] H_{i} \\
& +\frac{m_{d}}{v}\left[\bar{d}\left(\frac{\eta_{1}^{d} O_{\phi_{1} i}+\eta_{2}^{d} O_{\phi_{2} i}}{\eta_{1}^{d} c_{\beta}+\eta_{2}^{d} s_{\beta}}-i \frac{\eta_{1}^{d} s_{\beta}-\eta_{2}^{d} c_{\beta}}{\eta_{1}^{d} c_{\beta}+\eta_{2}^{d} s_{\beta}} O_{a i} \gamma_{5}\right) d\right] H_{i} \\
& +\frac{m_{l}}{v}\left[\bar{l}\left(\frac{\eta_{1}^{l} O_{\phi_{1} i}+\eta_{2}^{l} O_{\phi_{2} i}}{\eta_{1}^{l} c_{\beta}+\eta_{2}^{l} s_{\beta}}-i \frac{\eta_{1}^{l} s_{\beta}-\eta_{2}^{l} c_{\beta}}{\eta_{1}^{l} c_{\beta}+\eta_{2}^{l} s_{\beta}} O_{a i} \gamma_{5}\right) l\right] H_{i}
\end{aligned}
$$

and

$$
\begin{aligned}
-\mathcal{L}_{H^{ \pm} \bar{u} d}= & -\frac{\sqrt{2} m_{u}}{v}\left(\frac{c_{\beta}}{s_{\beta}}\right) \bar{u} P_{L} d H^{+}-\frac{\sqrt{2} m_{d}}{v}\left(\frac{\eta_{1}^{d} s_{\beta}-\eta_{2}^{d} c_{\beta}}{\eta_{1}^{d} c_{\beta}+\eta_{2}^{d} s_{\beta}}\right) \bar{u} P_{R} d H^{+} \\
& -\frac{\sqrt{2} m_{l}}{v}\left(\frac{\eta_{1}^{l} s_{\beta}-\eta_{2}^{l} c_{\beta}}{\eta_{1}^{l} c_{\beta}+\eta_{2}^{l} s_{\beta}}\right) \bar{\nu} P_{R} l H^{+}+\text {h.c. }
\end{aligned}
$$


Here we take the convention with $\xi=0$ and the couplings $h_{u, d, l}$ are supposed to be real. The $3 \times 3$ mixing matrix $O$ is defined through

$$
\left(\phi_{1}^{0}, \phi_{2}^{0}, a\right)_{\alpha}^{T}=O_{\alpha i}\left(H_{1}, H_{2}, H_{3}\right)_{i}^{T}
$$

such that $O^{T} \mathcal{M}_{0}^{2} O=\operatorname{diag}\left(M_{H_{1}}^{2}, M_{H_{2}}^{2}, M_{H_{3}}^{2}\right)$ with the ordering of $M_{H_{1}} \leq M_{H_{2}} \leq M_{H_{3}}$. Here the $3 \times 3$ mass matrix of the neutral Higgs bosons $\mathcal{M}_{0}^{2}$ is given by

$$
\mathcal{M}_{0}^{2}=M_{A}^{2}\left(\begin{array}{ccc}
s_{\beta}^{2} & -s_{\beta} c_{\beta} & 0 \\
-s_{\beta} c_{\beta} & c_{\beta}^{2} & 0 \\
0 & 0 & 1
\end{array}\right)+\mathcal{M}_{\lambda}^{2}
$$

with (reinstating the relative phase $\xi$ )

$$
\begin{aligned}
M_{A}^{2} & =M_{H^{ \pm}}^{2}+\frac{1}{2} \lambda_{4} v^{2}-\frac{1}{2} \Re \mathrm{e}\left(\lambda_{5} \mathrm{e}^{2 i \xi}\right) v^{2}, \\
M_{H^{ \pm}}^{2} & =\frac{\Re \mathrm{e}\left(m_{12}^{2} \mathrm{e}^{i \xi}\right)}{c_{\beta} s_{\beta}}-\frac{v^{2}}{2 c_{\beta} s_{\beta}}\left[\lambda_{4} c_{\beta} s_{\beta}+c_{\beta} s_{\beta} \Re \mathrm{e}\left(\lambda_{5} \mathrm{e}^{2 i \xi}\right)+c_{\beta}^{2} \Re \mathrm{e}\left(\lambda_{6} \mathrm{e}^{i \xi}\right)+s_{\beta}^{2} \Re \mathrm{e}\left(\lambda_{7} \mathrm{e}^{i \xi}\right)\right],
\end{aligned}
$$

and

$$
\frac{\mathcal{M}_{\lambda}^{2}}{v^{2}}=\left(\begin{array}{lll}
2 \lambda_{1} c_{\beta}^{2}+\Re \mathrm{e}\left(\lambda_{5} \mathrm{e}^{2 i \xi}\right) s_{\beta}^{2} & \lambda_{34} c_{\beta} s_{\beta}+\Re \mathrm{e}\left(\lambda_{6} \mathrm{e}^{i \xi}\right) c_{\beta}^{2} & -\frac{1}{2} \Im \mathrm{m}\left(\lambda_{5} \mathrm{e}^{2 i \xi}\right) s_{\beta} \\
+2 \Re \mathrm{e}\left(\lambda_{6} \mathrm{e}^{i \xi}\right) s_{\beta} c_{\beta} & +\Re \mathrm{e}\left(\lambda_{7} \mathrm{e}^{i \xi}\right) s_{\beta}^{2} & -\Im \mathrm{m}\left(\lambda_{6} \mathrm{e}^{i \xi}\right) c_{\beta} \\
\lambda_{34} c_{\beta} s_{\beta}+\Re \mathrm{e}\left(\lambda_{6} \mathrm{e}^{i \xi}\right) c_{\beta}^{2} & 2 \lambda_{2} s_{\beta}^{2}+\Re \mathrm{e}\left(\lambda_{5} \mathrm{e}^{2 i \xi}\right) c_{\beta}^{2} & -\frac{1}{2} \Im \mathrm{m}\left(\lambda_{5} \mathrm{e}^{2 i \xi}\right) c_{\beta} \\
+\Re \mathrm{e}\left(\lambda_{7} \mathrm{e}^{i \xi}\right) s_{\beta}^{2} & +2 \Re \mathrm{e}\left(\lambda_{7} \mathrm{e}^{i \xi}\right) s_{\beta} c_{\beta} & -\Im \mathrm{m}\left(\lambda_{7} \mathrm{e}^{i \xi}\right) s_{\beta} \\
-\frac{1}{2} \Im \mathrm{m}\left(\lambda_{5} \mathrm{e}^{2 i \xi}\right) s_{\beta} & -\frac{1}{2} \Im \mathrm{m}\left(\lambda_{5} \mathrm{e}^{2 i \xi}\right) c_{\beta} & 0 \\
-\Im \mathrm{m}\left(\lambda_{6} \mathrm{e}^{i \xi}\right) c_{\beta} & -\Im \mathrm{m}\left(\lambda_{6} \mathrm{e}^{i \xi}\right) s_{\beta} &
\end{array}\right)
$$

where $\lambda_{34}=\lambda_{3}+\lambda_{4}$ and, in passing, we note $v=g M_{W} / 2, a=-s_{\beta} a_{1}+c_{\beta} a_{2}$ and $H^{+}=-s_{\beta} \phi_{1}^{+}+c_{\beta} \phi_{2}^{+}$. We need to specify, therefore, the 13 parameters plus one sign listed in eq. (3.3) to fix all the Higgs-fermion-fermion couplings.

Nevertheless, in order to calculate the signal strengths on which our chi-square analysis is based, we need to know only the couplings of the $125 \mathrm{GeV}$ Higgs boson. Regarding the $i$-th Higgs boson $H_{i}$ as the candidate for the $125 \mathrm{GeV}$ Higgs boson, and by looking into eqs. (3.7) and (3.8), the relevant Higgs couplings can be fully determined by knowing the components $O_{\phi_{1} i}, O_{\phi_{2} i}$, and $O_{a i}$ of the mixing matrix and $t_{\beta}$ in each 2HDM. Comparing eqs. (3.7) and (2.1) we find

$$
\begin{aligned}
& O_{\phi_{2} i}=s_{\beta} C_{u}^{S}, \quad O_{a i}=-t_{\beta} C_{u}^{P} ; \\
& O_{\phi_{1} i}= \pm\left[1-s_{\beta}^{2}\left(C_{u}^{S}\right)^{2}-t_{\beta}^{2}\left(C_{u}^{P}\right)^{2}\right]^{1 / 2},
\end{aligned}
$$

where $C_{u}^{S}=g_{H_{i} \bar{u} u}^{S}$ and $C_{u}^{P}=g_{H_{i} \bar{u} u}^{P}$ and the orthogonality relation $\left(O_{\phi_{1} i}\right)^{2}+\left(O_{\phi_{2} i}\right)^{2}+$ $\left(O_{a i}\right)^{2}=1$ is used. ${ }^{1}$ Therefore, by specifying only the 3 parameters of $C_{u}^{S}, C_{u}^{P}$, and $t_{\beta}$, the

\footnotetext{
${ }^{1}$ Depending on the values of $\tan \beta, C_{u}^{S}$, and $C_{u}^{P}$, one may take one or both of the two signs for $O_{\phi_{1} i}$ by fixing the relative sign between the Yukawa and $g_{H_{i} V V}$ couplings. Without loss of generality we take the convention of $g_{H_{i} V V}=C_{v}>0$ in this work.
} 


\begin{tabular}{|l|c|c|c|c|}
\hline $2 \mathrm{HDM} \mathrm{I}$ & $C_{d}^{S}=C_{u}^{S}$ & $C_{l}^{S}=C_{u}^{S}$ & $C_{d}^{P}=-C_{u}^{P}$ & $C_{l}^{P}=-C_{u}^{P}$ \\
\hline $2 \mathrm{HDM} \mathrm{II}$ & $C_{d}^{S}= \pm \frac{\left[1-s_{\beta}^{2}\left(C_{u}^{S}\right)^{2}-t_{\beta}^{2}\left(C_{u}^{P}\right)^{2}\right]^{1 / 2}}{c_{\beta}}$ & $C_{l}^{S}= \pm \frac{\left[1-s_{\beta}^{2}\left(C_{u}^{S}\right)^{2}-t_{\beta}^{2}\left(C_{u}^{P}\right)^{2}\right]^{1 / 2}}{c_{\beta}}$ & $C_{d}^{P}=t_{\beta}^{2} C_{u}^{P}$ & $C_{l}^{P}=t_{\beta}^{2} C_{u}^{P}$ \\
\hline $2 \mathrm{HDM} \mathrm{III}$ & $C_{d}^{S}=C_{u}^{S}$ & $C_{l}^{S}= \pm \frac{\left[1-s_{\beta}^{2}\left(C_{u}^{S}\right)^{2}-t_{\beta}^{2}\left(C_{u}^{P}\right)^{2}\right]^{1 / 2}}{c_{\beta}}$ & $C_{d}^{P}=-C_{u}^{P}$ & $C_{l}^{P}=t_{\beta}^{2} C_{u}^{P}$ \\
\hline $2 \mathrm{HDM} \mathrm{IV}$ & $C_{d}^{S}= \pm \frac{\left[1-s_{\beta}^{2}\left(C_{u}^{S}\right)^{2}-t_{\beta}^{2}\left(C_{u}^{P}\right)^{2}\right]^{1 / 2}}{c_{\beta}}$ & $C_{l}^{S}=C_{u}^{S}$ & $C_{d}^{P}=t_{\beta}^{2} C_{u}^{P}$ & $C_{l}^{P}=-C_{u}^{P}$ \\
\hline
\end{tabular}

Table 2. The couplings $C_{d, l}^{S, P}$ as functions of $C_{u}^{S, P}$ and $\tan \beta$ in each 2HDM.

couplings of the $125 \mathrm{GeV}$ Higgs to all the SM fermions can be determined in each 2HDM as summarized in table 2. In addition, the Higgs coupling to the massive vector bosons is determined by

$$
C_{v}=c_{\beta} O_{\phi_{1} i}+s_{\beta} O_{\phi_{2} i}= \pm c_{\beta}\left[1-s_{\beta}^{2}\left(C_{u}^{S}\right)^{2}-t_{\beta}^{2}\left(C_{u}^{P}\right)^{2}\right]^{1 / 2}+s_{\beta}^{2} C_{u}^{S}
$$

To recapitulate, we need 13 parameters (plus one sign) to fix all the Higgs couplings to the SM particles and the Higgs boson spectrum fully in general 2HDMs. In contrast, only 3 parameters are needed for the couplings of the $125 \mathrm{GeV}$ Higgs candidate to the $\mathrm{SM}$ fermions and massive vector bosons. These 3 parameters are the two couplings of the $125 \mathrm{GeV}$ Higgs candidate to the scalar and pseudoscalar top-quark bilinears $\left(C_{u}^{S}\right.$ and $C_{u}^{P}$, respectively) and $\tan \beta$. One may use $C_{v}$ instead of $\tan \beta$ as shown later. In this work, we take advantage of the avenue with the smaller number of parameters to analyze the Higgs data.

With $C_{u}^{S}, C_{u}^{P}$, and $C_{v}$ (or $\tan \beta$ ) given, we also need to know the charged Higgs contribution to the Higgs coupling to two photons in order to calculate the signal strengths. The charged Higgs contribution to the Higgs coupling to two photons is given by

$$
\left(\Delta S_{i}^{\gamma}\right)^{H^{ \pm}}=-g_{H_{i} H^{+} H^{-}} \frac{v^{2}}{2 M_{H^{ \pm}}^{2}} F_{0}\left(\tau_{i H^{ \pm}}\right)
$$

where $\tau_{i H^{ \pm}}=M_{H_{i}}^{2} / 4 M_{H^{ \pm}}^{2}$ and $F_{0}(\tau)=\tau^{-1}\left[-1+\tau^{-1} f(\tau)\right]$ with

$$
f(\tau)=-\frac{1}{2} \int_{0}^{1} \frac{\mathrm{d} y}{y} \ln [1-4 \tau y(1-y)]= \begin{cases}\arcsin ^{2}(\sqrt{\tau}): & \tau \leq 1 \\ -\frac{1}{4}\left[\ln \left(\frac{\sqrt{\tau}+\sqrt{\tau-1}}{\sqrt{\tau}-\sqrt{\tau-1}}\right)-i \pi\right]^{2}: & \tau \geq 1\end{cases}
$$

The $g_{H_{i} H^{+} H^{-}}$coupling is defined in the interaction

$$
\mathcal{L}_{3 H}=v \sum_{i=1}^{3} g_{H_{i} H^{+} H^{-}} H_{i} H^{+} H^{-}
$$

with $g_{H_{i} H^{+} H^{-}}=\sum_{\alpha=\phi_{1}, \phi_{2}, a} O_{\alpha i} g_{\alpha H^{+} H^{-}}$. The effective couplings $g_{\alpha H^{+} H^{-}}$indeed involve all 
of the Higgs quartic couplings again and read [72-74]: ${ }^{2}$

$$
\begin{aligned}
g_{\phi_{1} H^{+} H^{-}}= & 2 s_{\beta}^{2} c_{\beta} \lambda_{1}+c_{\beta}^{3} \lambda_{3}-s_{\beta}^{2} c_{\beta} \lambda_{4}-s_{\beta}^{2} c_{\beta} \Re \mathrm{e} \lambda_{5}+s_{\beta}\left(s_{\beta}^{2}-2 c_{\beta}^{2}\right) \Re \mathrm{e} \lambda_{6} \\
& +s_{\beta} c_{\beta}^{2} \Re \mathrm{e} \lambda_{7}, \\
g_{\phi_{2} H^{+} H^{-}}= & 2 s_{\beta} c_{\beta}^{2} \lambda_{2}+s_{\beta}^{3} \lambda_{3}-s_{\beta} c_{\beta}^{2} \lambda_{4}-s_{\beta} c_{\beta}^{2} \Re \mathrm{e} \lambda_{5}+s_{\beta}^{2} c_{\beta} \Re \mathrm{e} \lambda_{6} \\
& +c_{\beta}\left(c_{\beta}^{2}-2 s_{\beta}^{2}\right) \Re \mathrm{e} \lambda_{7}, \\
g_{a H^{+} H^{-}}= & s_{\beta} c_{\beta} \Im \mathrm{m} \lambda_{5}-s_{\beta}^{2} \Im \mathrm{m} \lambda_{6}-c_{\beta}^{2} \Im \mathrm{m} \lambda_{7} .
\end{aligned}
$$

Therefore, in order to include $\left(\Delta S_{i}^{\gamma}\right)^{H^{ \pm}}$one may specify all the quartic couplings and the charged Higgs mass in principle, but, then, the situation goes back to the original case with 13 parameters plus one sign. Nevertheless, even in this case one can still keep the spirit of efficiency and simplicity by treating $\left(\Delta S_{i}^{\gamma}\right)^{H^{ \pm}}$itself as another free parameter in addition to the other three ones $C_{u}^{S}, C_{u}^{P}$ and $C_{v}$. And then, the results on $\left(\Delta S_{i}^{\gamma}\right)^{H^{ \pm}}$could be directly interpreted in terms of the coupling $g_{H_{i} H^{+} H^{-}}$of the $125 \mathrm{GeV}$ Higgs boson to the charged Higgses and the charged Higgs boson mass $M_{H^{ \pm}}$, as shown in eq. (3.15).

One caveat of our approach to analyze the Higgs data with only 3 or 4 parameters is that one cannot say much about the other two neutral Higgs bosons and the charged one which, in principle, can be either heavier or lighter than the candidate for the $125 \mathrm{GeV}$ Higgs. Before moving to the next section to present the results of various 2-, 3- and 4parameter fits, we would like to briefly comment on the status of experimental searches for the additional Higgs bosons.

At the LHC, both the ATLAS and CMS collaborations have searched for the additional neutral Higgses bosons up to $1 \mathrm{TeV}$ through their decays into two massive vector bosons, $H_{i} \rightarrow Z Z$ or $W W$ [75-78]. Without observing any positive signal, they put an upper bound on the relevant cross section $\sigma\left(p p \rightarrow H_{i} \rightarrow V V\right) .{ }^{3}$ The ATLAS collaboration performed the neutral Higgs-boson searches through the tau-lepton channel, $H_{i} \rightarrow \tau \tau$ [79]. While this applies for both the CP-even and CP-odd neutral Higgses up to $500 \mathrm{GeV}$, it was reported that the constraint for the additional CP-even Higgs from this channel is weaker than that from $H_{i} \rightarrow Z Z$ [80].

For the charged Higgs boson with mass around a few hundred $\mathrm{GeV}$, the strongest constrain may come from $\operatorname{BR}\left(\bar{B} \rightarrow X_{s} \gamma\right)$ through the additional loop contributions from the charged-Higgs bosons to the process $b \rightarrow s \gamma$ [80]. When the charged Higgs boson is lighter than the top quark, it can be searched at the LHC through the top-quark decay channel $t \rightarrow H^{+} b$ with the charged Higgs boson subsequently decaying into $c \bar{b}, c \bar{s}$, and $\tau^{+} \nu_{\tau}$. The direct searches of the charged Higgs boson at the LHC also set limits on the interactions of charged Higgs boson, but their constraints are still weaker than those from $\bar{B} \rightarrow X_{s} \gamma[80]$.

The current direct experimental searches for the additional Higgs bosons and their indirect effects on some flavor observables such as $\operatorname{BR}\left(\bar{B} \rightarrow X_{s} \gamma\right)$ should provide more

\footnotetext{
${ }^{2}$ Note the convention difference for $\lambda_{5}$ by a factor 2 .

${ }^{3}$ We note that,if $C P$ is conserved, the constraints provided by these search channels cannot be applied to the CP-odd state.
} 
stringent restrictions on the model parameters in addition to those obtained by fitting the $125-\mathrm{GeV}$ Higgs data only. This may deserve an independent study and we will discuss these crucial issues in detail in a future publication.

\section{Fits}

As shown in the previous section, the whole analysis of the couplings of the observed Higgs boson (denoted by $H_{i}$ ) in $2 \mathrm{HDM}$, including the $\mathrm{CP}$-conserving and CP-violating cases, can be performed with only 4 parameters: $C_{u}^{S}, C_{u}^{P}, C_{v},\left(\Delta S_{i}^{\gamma}\right)^{H^{ \pm}}$. In particular, we consider the following cases with respect to $\mathrm{CP}$-conserving or $\mathrm{CP}$-violating, and with/without charged Higgs contributions:

- $\mathrm{CP}$-conserving $(\mathrm{CPC})$ cases

- CPC2: $C_{u}^{S}, C_{v}$

- CPC3: $C_{u}^{S}, C_{v},\left(\Delta S^{\gamma}\right)^{H^{ \pm}}$

- CP-violating (CPV) cases

- CPV3: $C_{u}^{S}, C_{u}^{P}, C_{v}$

- CPV4: $C_{u}^{S}, C_{u}^{P}, C_{v},\left(\Delta S^{\gamma}\right)^{H^{ \pm}}$

Here $\mathbf{C P C}$ and $\mathbf{C P V}$ represent $\mathrm{CP}$-conserving and CP-violating fits, respectively, and the number denotes the number of varying parameters in each fit. In CPC2 and CPV3, the charged Higgs contribution $\left(\Delta S^{\gamma}\right)^{H^{ \pm}}=0$. Note that the varying parameters should satisfy the following relations due to the unitarity of the mixing matrix:

$$
s_{\beta}^{2}\left(C_{u}^{S}\right)^{2} \leq 1, \quad t_{\beta}^{2}\left(C_{u}^{P}\right)^{2} \leq 1, \quad s_{\beta}^{2}\left(C_{u}^{S}\right)^{2}+t_{\beta}^{2}\left(C_{u}^{P}\right)^{2} \leq 1 .
$$

One can use $\tan \beta$ in place of $C_{v}$ in the analysis by exploiting the relation derived from eq. (3.14):

$$
s_{\beta}^{2}=\frac{1-C_{v}^{2}}{1+\left(C_{u}^{S}\right)^{2}+\left(C_{u}^{P}\right)^{2}-2 C_{v} C_{u}^{S}},
$$

which is independent of $\operatorname{sign}\left[O_{\phi_{1} i}\right]$. When $C_{u}^{S}=1$ and $C_{u}^{P}=0$, the above relation becomes $s_{\beta}^{2}=\left(1+C_{v}\right) / 2$, which leads to $\tan \beta=\infty$ in the SM limit of $C_{v}=1$. On the other hand, as in many models beyond the $\mathrm{SM}$, if $C_{u}^{S}$ and/or $C_{u}^{P}$ deviate from its $\mathrm{SM}$ values 1 and 0 , respectively, one may end up in the opposite limit, $\tan \beta=0$, when the dynamics of the fit pushes $C_{v}$ to its maximally allowed value or 1 . In practice, one may wish to avoid the regions with small or (very) large $\tan \beta$ to maintain the perturbativity of the top and bottom Yukawa couplings $h_{t}$ and $h_{b}$, respectively. We therefore restrict the range of $\tan \beta$ between $10^{-4}$ and $10^{2}$.

Before presenting our numerical results, we briefly review the current Higgs data. Current Higgs data focus on a few decay channels of the Higgs boson: (i) $h \rightarrow \gamma \gamma$, (ii) $h \rightarrow Z Z^{*} \rightarrow \ell^{+} \ell^{-} \ell^{+} \ell^{-}$, (iii) $h \rightarrow W W^{*} \rightarrow \ell^{+} \bar{\nu} \ell^{-} \nu$, (iv) $h \rightarrow b \bar{b}$, and (v) $h \rightarrow \tau^{+} \tau^{-}$. 
We have used 22 data points in our analysis as in ref. [6]. To briefly summarize, the chi-square of all these 22 data points relative to the SM is

$$
18.94=7.89(\gamma \gamma: 6)+1.65\left(Z Z^{*}: 2\right)+3.70\left(W W^{*}: 5\right)+3.55(b \bar{b}: 4)+2.15\left(\tau^{+} \tau^{-}: 5\right),
$$

where the numbers in parentheses denote the number of data points in each decay mode. The chi-square per degree of freedom (dof) is about $18.94 / 22=0.86$ and the $p$-value is about $p_{\mathrm{SM}}=0.65$. We note the chi-square is dominated by the diphoton data with $\mu_{g g H+t t H}^{\mathrm{ATLAS}}=1.6 \pm 0.4$ and $\mu_{\text {untagged }}^{\mathrm{CMS}}=0.78_{-0.26}^{+0.28}$. Since the ATLAS data is about $1.5 \sigma$ larger than the SM while the CMS one is about $1 \sigma$ smaller, the dynamics of the fit cannot force the parameters to go into either direction.

\subsection{CP conserving fits}

In this subsection, we study the $\mathrm{CP}$-conserving case with $C_{u}^{P}=0$. In our numerical study, we find that $\tan \beta$ is bounded from above when $C_{u}^{S}$ deviates from its SM value 1 . Before presenting numerical results, we look into the correlation among the varying parameters $C_{u}^{S}, C_{v}$, and $\tan \beta$.

In the CP-conserving case, eq. (3.14) simplifies into

$$
C_{v}= \pm c_{\beta}\left[1-s_{\beta}^{2}\left(C_{u}^{S}\right)^{2}\right]^{1 / 2}+s_{\beta}^{2} C_{u}^{S},
$$

with the constraint $\left|s_{\beta} C_{u}^{S}\right| \leq 1$, which can be recast into the form

$$
-\frac{1}{t_{\beta}} \leq C_{u}^{S} \leq \sqrt{1+\frac{1}{t_{\beta}^{2}}},
$$

taking into account our convention of $C_{v}>0$. For a given value of $\tan \beta$, we find that $C_{v}$ takes the plus $(+) \operatorname{sign}$ as $C_{u}^{S}$ increases from $-1 / t_{\beta}\left(\right.$ where $\left.C_{v}=0\right)$ to $\sqrt{1+1 / t_{\beta}^{2}}$. While it takes the minus (-) sign when $C_{u}^{S}$ goes from the maximum value $\sqrt{1+1 / t_{\beta}^{2}}$ back to $1 / t_{\beta}$ where again $C_{v}=0$. Therefore, $C_{v}$ has two positive solutions if $C_{u}^{S}$ lies between $1 / t_{\beta}$ and $\sqrt{1+1 / t_{\beta}^{2}}$. This behavior is shown in the left frame of figure 1. From eq. (4.2) which now can be rearranged into the form

$$
s_{\beta}^{2}=\frac{\left(1-C_{v}^{2}\right)}{\left(1-C_{v}^{2}\right)+\left(C_{u}^{S}-C_{v}\right)^{2}},
$$

we can see that $\sin \beta=1$ or $\tan \beta=\infty$ along the line $C_{v}=C_{u}^{S}$. Also, the larger $\tan \beta$ the smaller $C_{v}$ will be. Therefore, $\tan \beta$ will be bounded from above when $C_{v}$ is pushed to be close to 1 , unless $C_{u}^{S}=1$.

To be more precise, we consider the situation in which $C_{v}$ is constrained as $C_{v}>$ $\left(C_{v}\right)_{\min }$. As illustrated in the right frame of figure 1 with three values of $C_{u}^{S}=0.9$ (black), 1 (red), and 1.1 (blue), we have found that $\tan \beta$ has an upper bound when $C_{u}^{S}<\left(C_{v}\right)_{\min }$ for $C_{u}^{S}<1$. We observe that the upper bound on $\tan \beta$ is stronger when $\left(C_{v}\right)_{\min }$ is closer to 1 but it disappears when $\left(C_{v}\right)_{\min }<C_{u}^{S}$ or $C_{u}^{S}=1$. On the other hand, when $C_{u}^{S}>1$, 

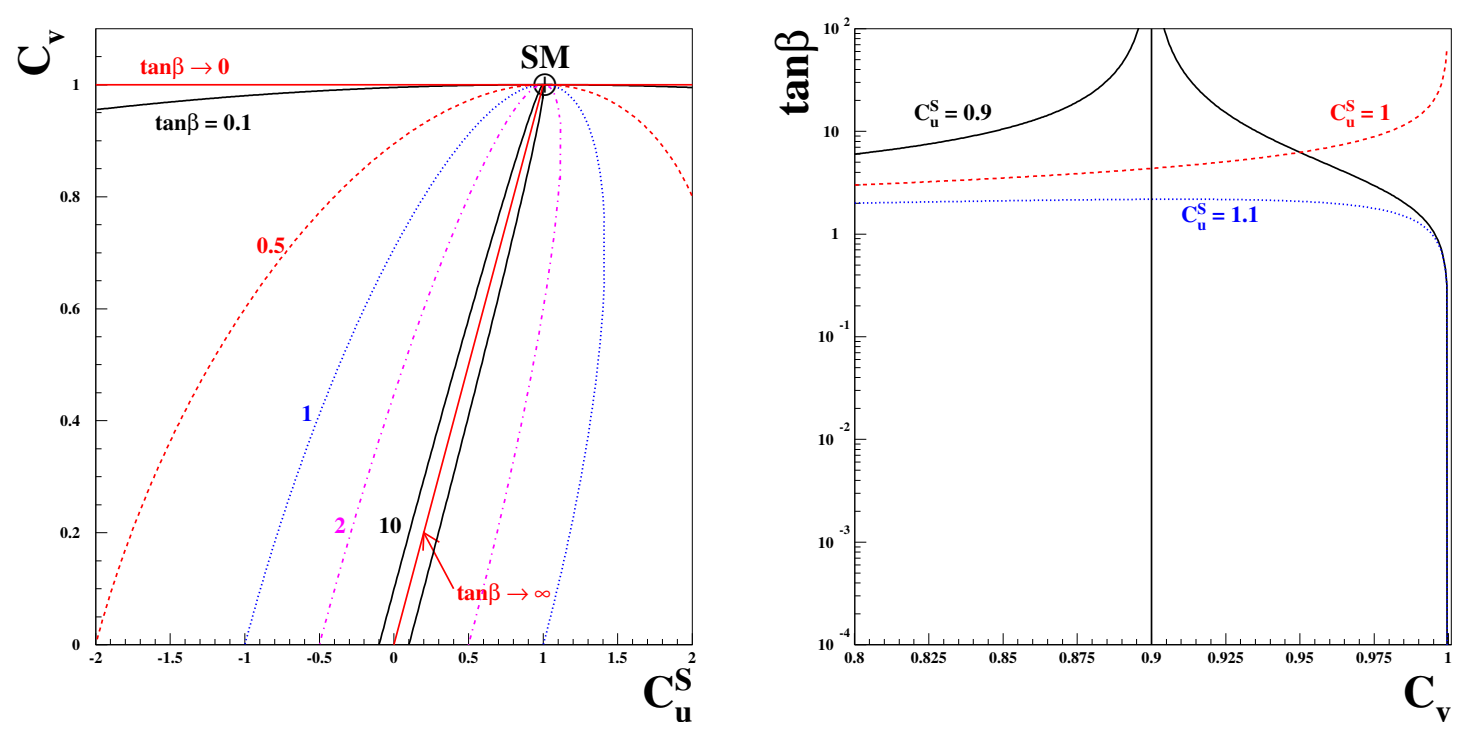

Figure 1. (Left) $C_{v}$ as functions of $C_{u}^{S}$ for several values of $\tan \beta=0.1$ (black) 0.5 (red), 1 (blue), 2 (magenta), and 10 (black). The horizontal red line is for the limit $\tan \beta \rightarrow 0$ and the straight red line with $C_{v}=C_{u}^{S}$ represents the limits $\tan \beta \rightarrow \infty$. The SM point with $C_{v}=C_{u}^{S}=1$ is denoted by $\oplus$. (Right) $\tan \beta$ as functions $C_{v}$ for three values of $C_{u}^{S}=0.9$ (black), 1 (red), and 1.1 (blue). The vertical line shows the location $C_{v}=0.9$.

$\tan \beta$ is always bounded by $\tan \beta \leq 1 / \sqrt{\left(C_{u}^{S}\right)^{2}-1}$, see eq. (4.3). Requiring $C_{v}>0.95$, for example, we find $\tan \beta \lesssim 6$ for $C_{u}^{S}=0.9$ and $\tan \beta \lesssim 1 / \sqrt{\left(C_{u}^{S}\right)^{2}-1} \simeq 2$ for $C_{u}^{S}=1.1$.

In the following sub-subsections, we illustrate that the precise and independent measurements of $C_{u}^{S}$ and $\tan \beta$ can tell us the phenomenological viability of 2 HDMs and/or enable us to make discrimination among them.

The results for various fits (CPC2, CPC3, CPV3, and CPV4) are tabulated in tables 3 and 4, and confidence regions are shown in figures 2-21.

\subsubsection{CPC2}

The fit CPC2 analyzes the Higgs data by varying $C_{u}^{S}$ and $C_{v}$ (or equivalently $\log _{10} \tan \beta$ ). The total $\chi^{2}, \chi^{2} /$ dof, $p$-value and the best-fit values of $C_{u}^{S}, C_{v}$, and $\tan \beta$ for the types I-IV of $2 \mathrm{HDM}$ are shown at the top of table 3 . We have found that the type-I model gives the smallest $\chi^{2}$ but the variation of total $\chi^{2}$ among the 4 types is very small, within 0.29 . Statistically, there is no preference among any type I to IV of $2 \mathrm{HDMs}$. We note that the $p$-values of the fits are all worse than the SM one $p_{\mathrm{SM}}=0.65$. The best-fit values for $C_{u}^{S}$ are about 0.9 for type I and III, and about 0.96 for type II and IV. The fitted $C_{v}$ 's are very close to the theoretically allowed maximum value 1 independent of the type. In the actual implementation, we used $\log _{10} \tan \beta$ as the scanning variable with $-4<\log _{10} \tan \beta<2$, instead of $C_{v}$. Again, independent of the type, $\chi^{2}$ continues to decrease as $\tan \beta$ falls below its lower limit $\tan \beta=10^{-4}$, though extremely slowly. The best fitted values for $\tan \beta$ are denoted by limit in table 3 . 


\begin{tabular}{|rc|rcc|rrrrr|}
\hline Fits & Type & $\chi^{2}$ & $\chi^{2} /$ dof & $p$-value & \multicolumn{5}{|c|}{ Best-fit values } \\
& & & & & $C_{u}^{S}$ & $C_{u}^{P}$ & $C_{v}$ & $\tan \beta$ & $\left(\Delta S^{\gamma}\right)^{H^{ \pm}}$ \\
\hline \multirow{3}{*}{ CPC2 } & I & 18.39 & 0.920 & 0.562 & 0.895 & 0 & 1.000 & limit & 0 \\
& II & 18.68 & 0.934 & 0.543 & 0.963 & 0 & 1.000 & limit & 0 \\
& III & 18.44 & 0.922 & 0.558 & 0.892 & 0 & 1.000 & limit & 0 \\
& IV & 18.66 & 0.933 & 0.544 & 0.965 & 0 & 1.000 & limit & 0 \\
\hline \multirow{6}{*}{ CPC3 } & I & 17.64 & 0.928 & 0.547 & 0.924 & 0 & 0.965 & 6.308 & -0.756 \\
& I & 17.64 & 0.928 & 0.547 & -0.921 & 0 & 0.965 & 0.144 & 2.377 \\
& II & 17.30 & 0.910 & 0.570 & -0.822 & 0 & 1.000 & $2 \times 10^{-4}$ & 2.218 \\
& III & 17.63 & 0.928 & 0.547 & -0.912 & 0 & 0.967 & 0.137 & 2.365 \\
& IV & 17.54 & 0.923 & 0.553 & 0.955 & 0 & 1.000 & 0.662 & -0.835 \\
\hline \hline \multirow{6}{*}{ CPV3 } & I & 18.37 & 0.967 & 0.498 & 0.867 & 0.142 & 0.988 & 0.840 & 0 \\
& I & 18.37 & 0.967 & 0.498 & 0.867 & -0.142 & 0.988 & 0.840 & 0 \\
& II & 17.17 & 0.904 & 0.578 & 0.476 & -0.505 & 0.998 & 0.082 & 0 \\
& II & 17.17 & 0.904 & 0.578 & 0.475 & 0.505 & 0.998 & 0.095 & 0 \\
& III & 18.41 & 0.969 & 0.495 & 0.873 & -0.110 & 1.000 & $2 \times 10^{-4}$ & 0 \\
& III & 18.41 & 0.969 & 0.495 & 0.873 & 0.109 & 1.000 & $1.2 \times 10^{-4}$ & 0 \\
& IV & 18.16 & 0.956 & 0.512 & 0.806 & 0.339 & 1.000 & limit & 0 \\
& IV & 18.16 & 0.956 & 0.512 & 0.806 & -0.339 & 1.000 & $1.2 \times 10^{-4}$ & 0 \\
\hline & I & 17.64 & 0.980 & 0.480 & 0.924 & $-1.5 \times 10^{-3}$ & 0.964 & 6.488 & -0.777 \\
& I & 17.64 & 0.980 & 0.480 & -0.924 & $2 \times 10^{-4}$ & 0.965 & 0.139 & 2.389 \\
CPV4 & II & 17.07 & 0.948 & 0.518 & -0.052 & 0.572 & 0.999 & 0.045 & 1.042 \\
& II & 17.07 & 0.948 & 0.518 & -0.052 & -0.572 & 0.999 & 0.045 & 1.042 \\
& III & 17.64 & 0.980 & 0.480 & -0.909 & 0.032 & 0.972 & 0.126 & 2.370 \\
& IV & 17.54 & 0.975 & 0.486 & 0.956 & -0.016 & 1.000 & 0.670 & -0.831 \\
\hline
\end{tabular}

Table 3. The best-fit values for various $\mathrm{CPC}$ and CPV fits. The SM values are: $\chi^{2}=18.94$, $\chi^{2} /$ dof $=0.86$, and $p$-value $=0.65$.

We show the contour plots for confidence-level regions as functions $C_{u}^{S}$ vs $C_{v}, C_{u}^{S}$ vs $\tan \beta$, and $C_{d}^{S}$ vs $C_{l}^{S}$ in figures $2-4$, respectively. The regions shown are for $\Delta \chi^{2} \leq 2.3$ (red), 5.99 (green), and 11.83 (blue) above the minimum, which correspond to confidence levels of $68.3 \%, 95 \%$, and $99.7 \%$, respectively. The best-fit point is denoted by the triangle. We note that from figure 2 there are two islands and positive $C_{u}^{S}$ is preferred. At $99.7 \%$ confidence level (CL), $C_{v} \gtrsim 0.7$. We also find that $C_{u}^{S}$ takes on the values between 0.71 and 1.2 (I), 0.86 and 1.1 (II), 0.71 and 1.2 (III), and 0.86 and 1.1 (IV) at 68.3\% CL. Comparing type I with the other three types, we find that the preference for $C_{u}^{S}=1$ is stronger in type II, III, and IV, and $C_{v}$ is more strongly constrained to be close to 1 unless $C_{u}^{S}=1$. Furthermore, the $\tan \beta=\infty$ line with $C_{u}^{S}=C_{v}$ passes through the CL regions only in type I.

In figure 3, we show the CL regions in the plane of $C_{u}^{S}$ and $\tan \beta$. For $\tan \beta \lesssim 0.5$, we find $\chi^{2}$ is almost independent of $\tan \beta$ for a fixed value of $C_{u}^{S}$; while for $\tan \beta \gtrsim 1$, the values of $C_{u}^{S}$ is constrained by $C_{u}^{S} \leq \sqrt{1+1 / t_{\beta}^{2}}$. For type I, as we observed in figure 2 , 

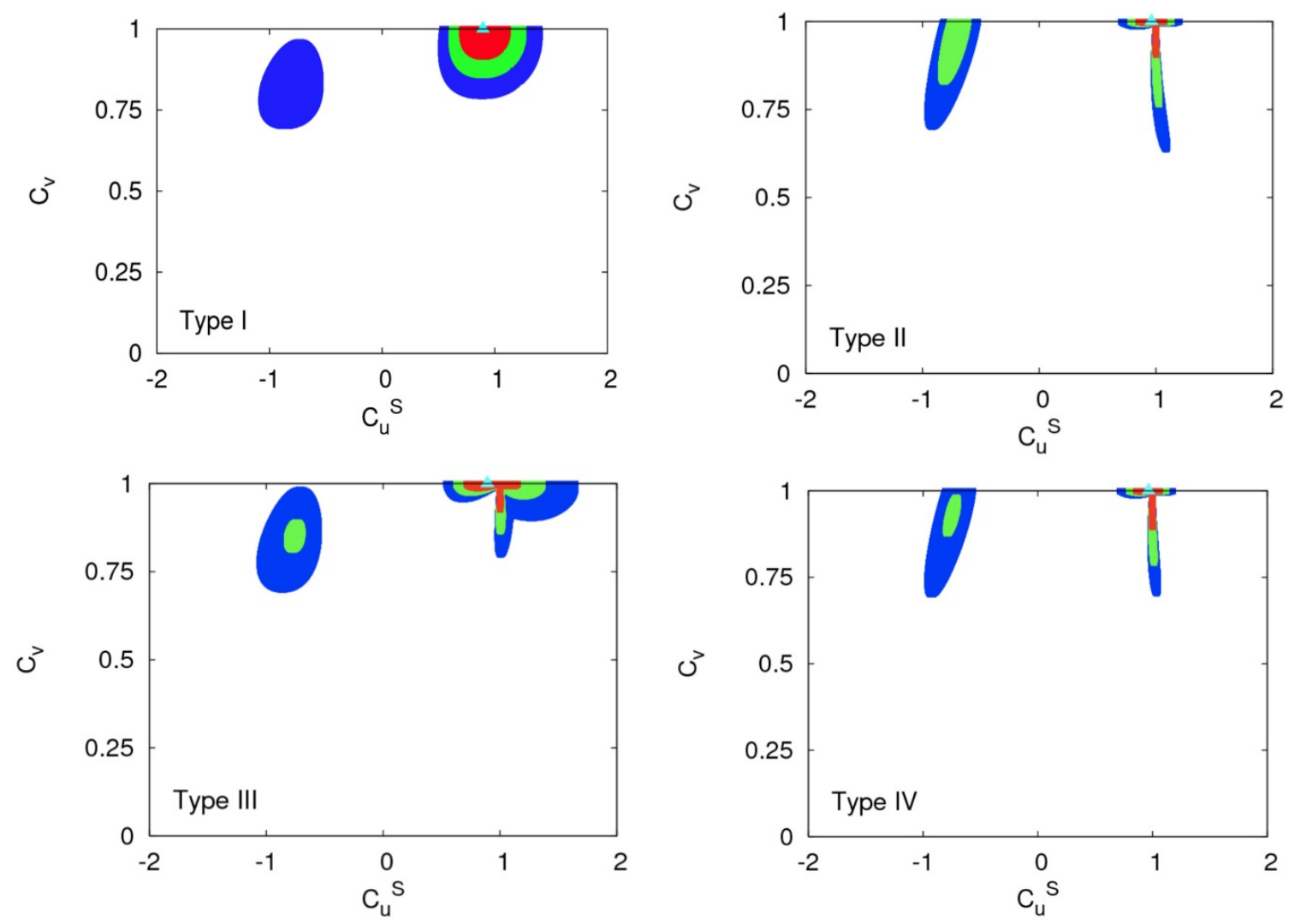

Figure 2. The confidence-level regions of the fit by varying $C_{u}^{S}$ and $C_{v}$ (or equivalently $\log _{10} \tan \beta$ ) only (CPC2 case) in the plane of $C_{u}^{S}$ vs $C_{v}$ for Type I-IV. The contour regions shown are for $\Delta \chi^{2} \leq 2.3$ (red), 5.99 (green), and 11.83 (blue) above the minimum, which correspond to confidence levels of $68.3 \%, 95 \%$, and $99.7 \%$, respectively. The best-fit points are denoted by the triangle.

the $\tan \beta=\infty$ line passes through the CL regions and it explains why we can have very large $\tan \beta$ in relatively broader range of $C_{u}^{S}$. For the other three types it is only possible to have very large $\tan \beta$ in the narrow region around $C_{u}^{S}=1$. Thus, in these cases we find that $\tan \beta \lesssim 3$ (II), 2 (III), 3 (IV) at $99.7 \%$ CL when the best-fit value of $C_{u}^{S}$ is taken in each of the type II, III, and IV. If precise and independent measurements of $C_{u}^{S}$ and $\tan \beta$ are available in future experiments, one can tell the phenomenological viability of $2 \mathrm{HDMs}$. For example, if $\tan \beta \gtrsim 10$ and $C_{u}^{S} \neq 1$, then one can rule out the type II, III, and IV models based on figure 3 .

In figure 4, we show the CL regions in the plane of $C_{d}^{S}$ and $C_{l}^{S}$. From table 2, the following relations $C_{d}^{S}=C_{l}^{S}=C_{u}^{S}$ (I), $C_{d}^{S}=C_{l}^{S}$ (II), $C_{d}^{S}=C_{u}^{S}$ (III), and $C_{l}^{S}=C_{u}^{S}$ (IV) are hold. In table 4 , we can see that the best-fit values of $C_{d}^{S}$ and/or $C_{l}^{S}$ are +1 unless either or both of them are equal to $C_{u}^{S}$. This can be understood from the relation, for example in type II,

$$
C_{d}^{S}=C_{l}^{S}=\frac{\sqrt{1-s_{\beta}^{2}\left(C_{u}^{S}\right)^{2}}}{c_{\beta}}=\sqrt{1+t_{\beta}^{2}\left[1-\left(C_{u}^{S}\right)^{2}\right]}
$$



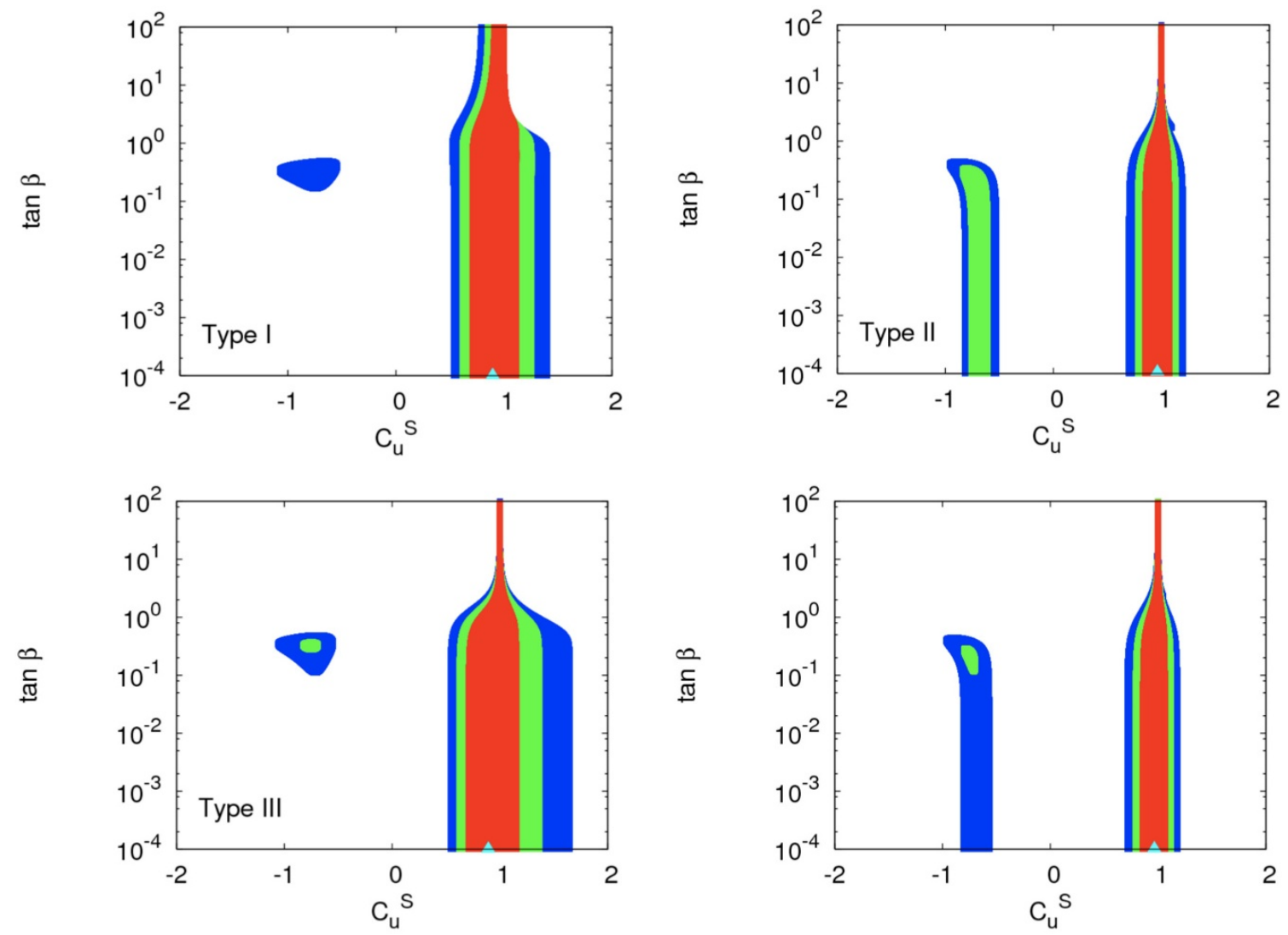

Figure 3. The same as figure 2 but in the plane of $C_{u}^{S}$ vs $\tan \beta$ (CPC2). The description of the confidence regions is the same as figure 2 .

with the best-fit values of $C_{u}^{S}=0.963$ and $\tan \beta=$ limit $=10^{-4}$. Note that the positive sign is selected to explain the best-fit values of $C_{d, l}^{S}$. Taking into account the negative sign, we observe that the points around $\left(C_{d}^{S}, C_{l}^{S}\right)=(-1,-1)(\mathrm{II}),\left(C_{d}^{S}, C_{l}^{S}\right)=(+1,-1)$ (III), and $\left(C_{d}^{S}, C_{l}^{S}\right)=(-1,+1)(\mathrm{IV})$ are also allowed at $68.3 \%$ CL even when $C_{u}^{S}$ is positive.

So far in this CPC2 fit we only found very small $\chi^{2}$ differences among the four types. What if the discrete symmetries are relaxed, do we get a better $\chi^{2}$ fit? We relax the requirement on the discrete symmetries, which enforces $\eta_{1,2}^{d, \ell}$ to be either 0 or 1 , but still require $\left(\eta_{1}^{d, \ell}\right)^{2}+\left(\eta_{2}^{d, \ell}\right)^{2}=1$. We therefore have two more free parameters in our scan, and they are $\eta_{1}^{d, \ell}$, leading to a four-parameter fit by varying $C_{u}^{S}, C_{v}, \eta_{1}^{d}$, and $\eta_{1}^{\ell}$. In figure 5 , we show the CL regions of the fit by varying $C_{u}^{S}, C_{v}, \eta_{1}^{d}$, and $\eta_{1}^{\ell}$ in the plane of $\eta_{1}^{d}$ and $\eta_{1}^{\ell}{ }^{4}$ We observe that $\Delta \chi^{2}<1$ in the whole $\left(\eta_{1}^{d}, \eta_{1}^{\ell}\right)$ plane, and so conclude that one cannot say any preference based on the current Higgs data.

\subsubsection{CPC3}

In this CPC3 fit, we vary three parameters: $C_{u}^{S}, C_{v}$ (or equivalently $\log _{10} \tan \beta$ ), and $\left(\Delta S^{\gamma}\right)^{H^{ \pm}}$. The total $\chi^{2}, \chi^{2} /$ dof, $p$-value and the best-fit values of $C_{u}^{S}, C_{v}(\tan \beta)$, and

\footnotetext{
${ }^{4}$ We obtain the minimum $\chi^{2}=18.30$ and $\chi^{2} /$ dof $=1.02$ for this fit.
} 

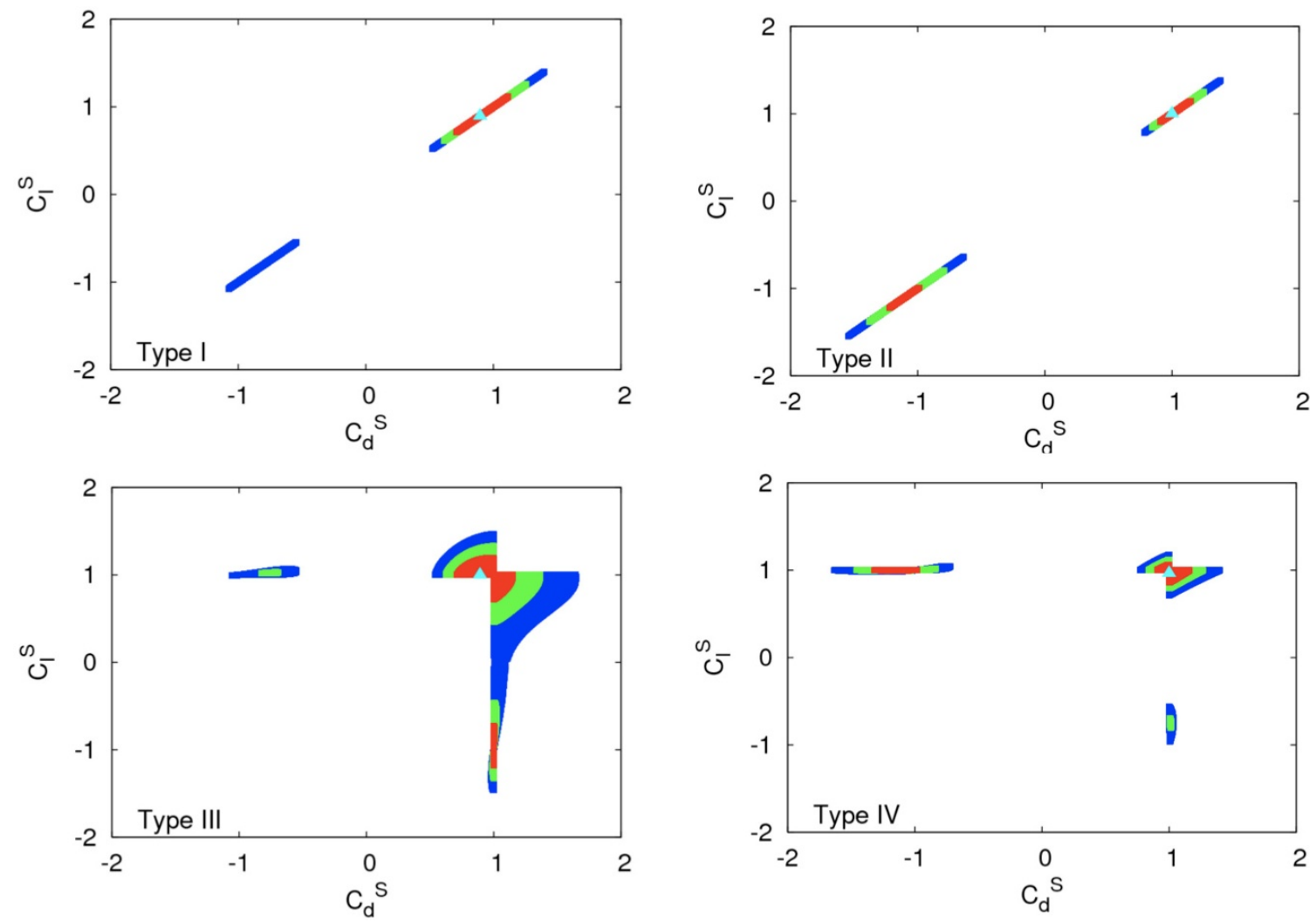

Figure 4. The same as figure 2 but in the plane of $C_{d}^{S}$ vs $C_{\ell}^{S}$ (CPC2). The description of the confidence regions is the same as figure 2 .

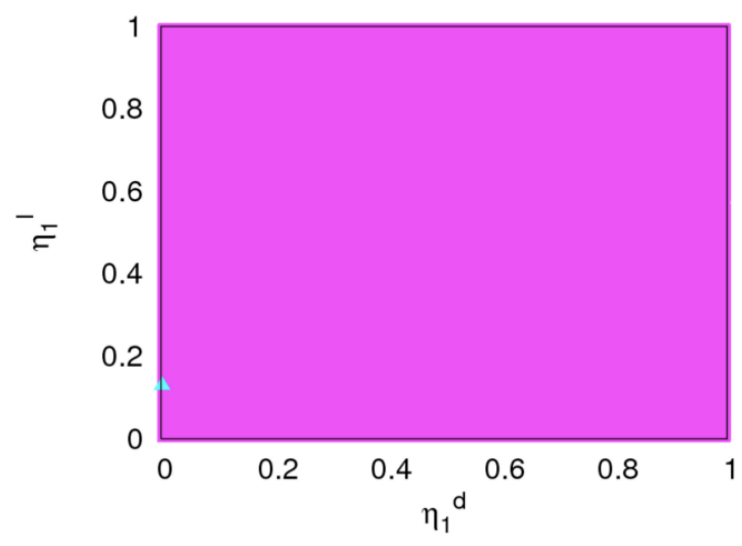

Figure 5. The confidence-level regions of the fit by varying $C_{u}^{S}, C_{v}, \eta_{1}^{d}$, and $\eta_{1}^{\ell}$ in the plane of $\eta_{1}^{d}$ vs $\eta_{1}^{\ell}$. The best-fit points are denoted by the triangle. Here the entire region is for $\Delta \chi^{2}<1.0$.

$\left(\Delta S^{\gamma}\right)^{H^{ \pm}}$for the types I-IV of 2 HDMs are shown in the upper half of table 3. We show the contour plots for confidence-level regions as functions $C_{u}^{S}$ vs $C_{v}, C_{u}^{S}$ vs $\tan \beta, C_{u}^{S}$ vs $\left(\Delta S^{\gamma}\right)^{H^{ \pm}}$, and $C_{d}^{S}$ vs $C_{l}^{S}$ in figures $6-9$, respectively.

We found that type II gives the smallest $\chi^{2}$ but the variation of total $\chi^{2}$ among the four types is very small, within 0.34. The CPC3 fit is slightly better than the CPC2, as it has 


\begin{tabular}{|cc|rcc|rrrr|}
\hline Fits & Type & $\chi^{2}$ & $\chi^{2} /$ dof & $p$-value & \multicolumn{4}{|c|}{ Best-fit values } \\
& & & & & $C_{d}^{S}$ & $C_{l}^{S}$ & $C_{d}^{P}$ & $C_{l}^{P}$ \\
\hline \multirow{2}{*}{ CPC2 } & I & 18.39 & 0.920 & 0.562 & 0.896 & 0.896 & 0 & 0 \\
& II & 18.68 & 0.934 & 0.543 & 1.000 & 1.000 & 0 & 0 \\
& III & 18.44 & 0.922 & 0.558 & 0.892 & 1.000 & 0 & 0 \\
& IV & 18.66 & 0.933 & 0.544 & 1.000 & 0.965 & 0 & 0 \\
\hline & I & 17.64 & 0.928 & 0.547 & 0.923 & 0.923 & 0 & 0 \\
& I & 17.64 & 0.928 & 0.547 & -0.923 & -0.923 & 0 & 0 \\
CPC3 & II & 17.30 & 0.910 & 0.570 & 1.000 & 1.000 & 0 & 0 \\
& III & 17.63 & 0.928 & 0.547 & -0.914 & 1.002 & 0 & 0 \\
& IV & 17.54 & 0.923 & 0.553 & 1.015 & 0.951 & 0 & 0 \\
\hline \hline & I & 18.37 & 0.967 & 0.498 & 0.867 & 0.867 & -0.142 & -0.142 \\
& I & 18.37 & 0.967 & 0.498 & 0.867 & 0.867 & 0.142 & 0.142 \\
CPV3 & II & 17.17 & 0.904 & 0.578 & 1.002 & 1.002 & $-4.6 \times 10^{-3}$ & $-4.6 \times 10^{-3}$ \\
& II & 17.17 & 0.904 & 0.578 & 1.002 & 1.002 & $4.6 \times 10^{-3}$ & $4.6 \times 10^{-3}$ \\
& III & 18.41 & 0.969 & 0.495 & 0.873 & 1.000 & 0.109 & 0 \\
& III & 18.41 & 0.969 & 0.495 & 0.873 & 1.000 & -0.109 & 0 \\
& IV & 18.16 & 0.956 & 0.512 & 1.000 & 0.806 & 0 & -0.339 \\
& IV & 18.16 & 0.956 & 0.512 & 1.000 & 0.806 & 0 & 0.339 \\
\hline & I & 17.64 & 0.980 & 0.480 & 0.924 & 0.924 & $1.5 \times 10^{-3}$ & $1.5 \times 10^{-3}$ \\
& I & 17.64 & 0.980 & 0.480 & -0.924 & -0.924 & $-2 \times 10^{-4}$ & $-2 \times 10^{-4}$ \\
CPV4 & II & 17.07 & 0.948 & 0.518 & 1.001 & 1.001 & $1.2 \times 10^{-3}$ & $1.2 \times 10^{-3}$ \\
& II & 17.07 & 0.948 & 0.518 & 1.001 & 1.001 & $-1.2 \times 10^{-3}$ & $-1.2 \times 10^{-3}$ \\
& III & 17.64 & 0.980 & 0.480 & -0.914 & 1.002 & $-3 \times 10^{-5}$ & 0 \\
& IV & 17.54 & 0.975 & 0.486 & 1.015 & 0.951 & $-1 \times 10^{-3}$ & $3 \times 10^{-3}$ \\
\hline
\end{tabular}

Table 4. Table showing the corresponding best-fit values for $C_{d, l}^{S, P}$.

one more parameter in the fit. However, the $p$-values of the fits are still worse than the SM one $\left(p_{\mathrm{SM}}=0.65\right)$. The best-fit values for $C_{u}^{S}$ are about $\pm 0.92(\mathrm{I}),-0.82$ (II), -0.91 (III), and 0.96 (IV) and those of $C_{v}$ are 0.97 for I and III and 1 for II and IV. We also implement independent fits with $\log _{10} \tan \beta$ as the scanning variable taking $-4<\log _{10} \tan \beta<2$, instead of $C_{v}$. The best-fit values for $\tan \beta$ are either small or very small, except for type I with positive $C_{u}^{S}$. Again, we note that $\chi^{2}$ hardly changes as $\tan \beta$ varies in wide range of parameter space.

For $\left(\Delta S^{\gamma}\right)^{H^{ \pm}}$, we have obtained $\left(\Delta S^{\gamma}\right)^{H^{ \pm}} \simeq-0.8$ or 2.3 when $C_{u}^{S} \sim+0.9$ or -0.9 , respectively. This can be understood from the numerical expression for $S^{\gamma}$ [6]

$$
S^{\gamma} \simeq-8.35 C_{v}+1.76 C_{u}^{S}+\left(\Delta S^{\gamma}\right)^{H^{ \pm}} .
$$

When $C_{u}^{S}$ changes from +0.9 to $-0.9,\left(\Delta S^{\gamma}\right)^{H^{ \pm}}$changes from -0.8 to +2.3 so that the sum $1.76 C_{u}^{S}+\left(\Delta S^{\gamma}\right)^{H^{ \pm}} \approx 0.7$.

The contour plots for the CL regions in the plane of $C_{u}^{S}$ vs $C_{v}$ for type I-IV are shown in figure 6 , which can be directly compared to figure 2 . In contrast, the negative $C_{u}^{S}$ is now 

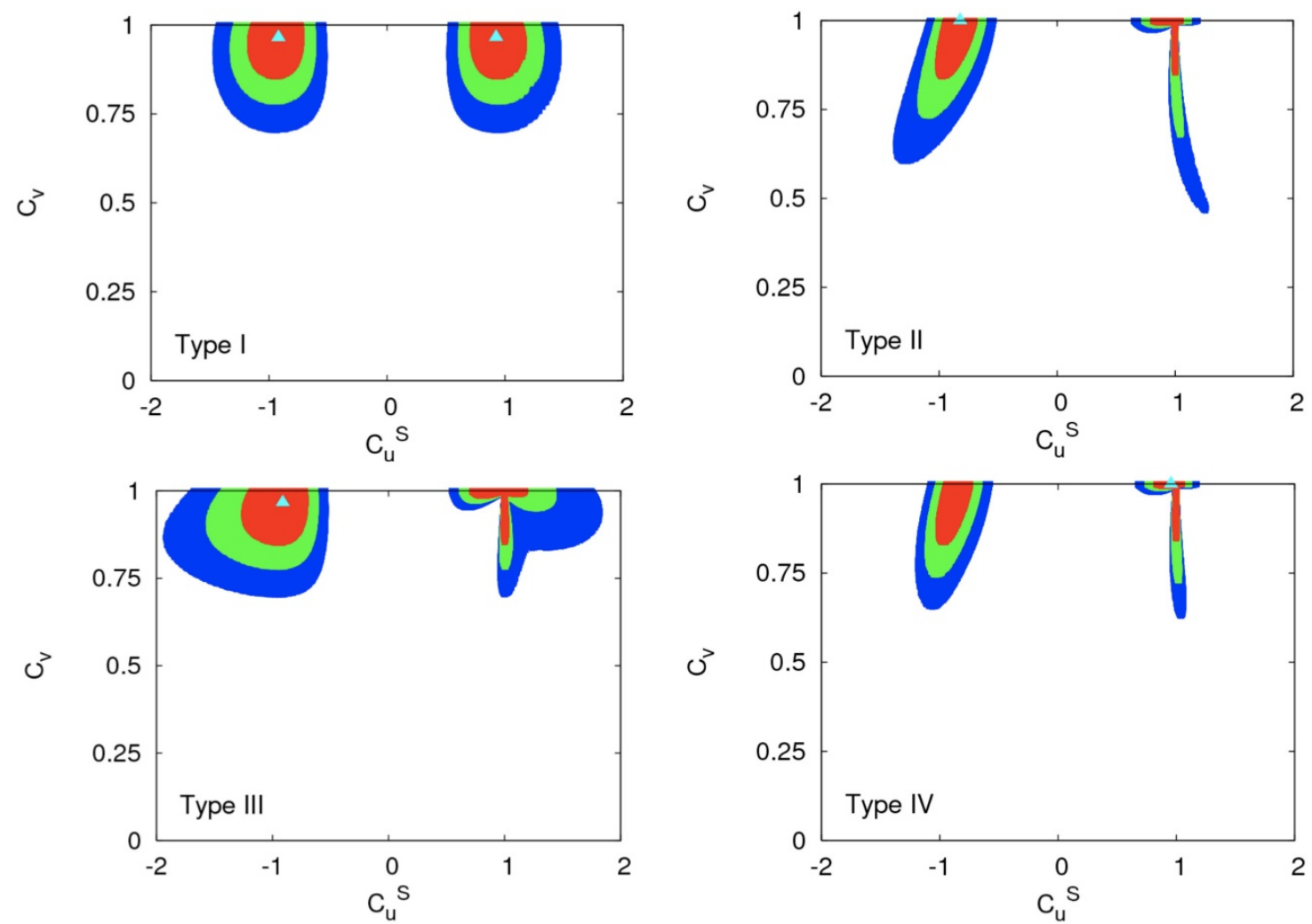

Figure 6. The confidence-level regions of the fit by varying $C_{u}^{S}, \log _{10} \tan \beta$, and $\Delta S^{\gamma}$ (CPC3 case) in the plane of $C_{u}^{S}$ vs $C_{v}$ for Type I-IV. The contour regions shown are for $\Delta \chi^{2} \leq 2.3$ (red), 5.99 (green), and 11.83 (blue) above the minimum, which correspond to confidence levels of $68.3 \%$, $95 \%$, and $99.7 \%$, respectively. The best-fit points are denoted by the triangle.

equally as good as the positive one. We show the CL regions in the plane of $C_{u}^{S}$ and $\tan \beta$ in figure 7. For the negative $C_{u}^{S}$ case, we find that $\tan \beta$ is smaller than $\sim 0.6$ at 99.7\% CL. In figure 8, we show the CL regions in the plane of $C_{u}^{S}$ and $\left(\Delta S^{\gamma}\right)^{H^{ \pm}}$. For positive $C_{u}^{S}$, it lies between 2 and -4 while $\left(\Delta S^{\gamma}\right)^{H^{ \pm}}>-0.7 \sim-1.6$ for negative $C_{u}^{S}$ at $99.7 \% \mathrm{CL}$. The CL regions for $C_{l}^{S}$ and $C_{d}^{S}$ are similar to the CPC2 case as shown in figure 9 but with the larger regions allowed at $68.5 \% \mathrm{CL}$ around the negative values of couplings.

The single parameter $\left(\Delta S^{\gamma}\right)^{H^{ \pm}}$can be interpreted in terms of the charged Higgs mass $M_{H^{ \pm}}$and the neutral Higgs coupling to the charged Higgses $g_{H_{i} H^{+} H^{-}}$, as in eq. (3.15). In figure 10, we show the CL regions in the plane of $M_{H^{ \pm}}$vs $g_{H_{i} H^{+} H^{-}}$. Since the variation of $\chi^{2}$ is very mild, we add one more region with $\Delta \chi^{2} \leq 1$ (black). The thick cyan lines denote the points giving the best-fit values of $\left(\Delta S^{\gamma}\right)^{H^{ \pm}}$in each type given by eq. (3.15). We see that a smaller charged Higgs mass is preferred when $g_{H_{i} H^{+} H^{-}}<0$, because this corresponds to $C_{u}^{S}<0$ and so a larger $\left(\Delta S^{\gamma}\right)^{H^{ \pm}} \approx 2.3$ is required. If the charged Higgs mass is larger than $\sim 300 \mathrm{GeV}$ as in the type II model constrained by $B(b \rightarrow s \gamma)$, we can see that the positive $C_{u}^{S}$ case with $\left(\Delta S^{\gamma}\right)^{H^{ \pm}} \sim-0.8$ is somewhat preferred. Nevertheless, the variation of $\chi^{2}$ is not large enough to have a conclusive statement based on the current Higgs data. 

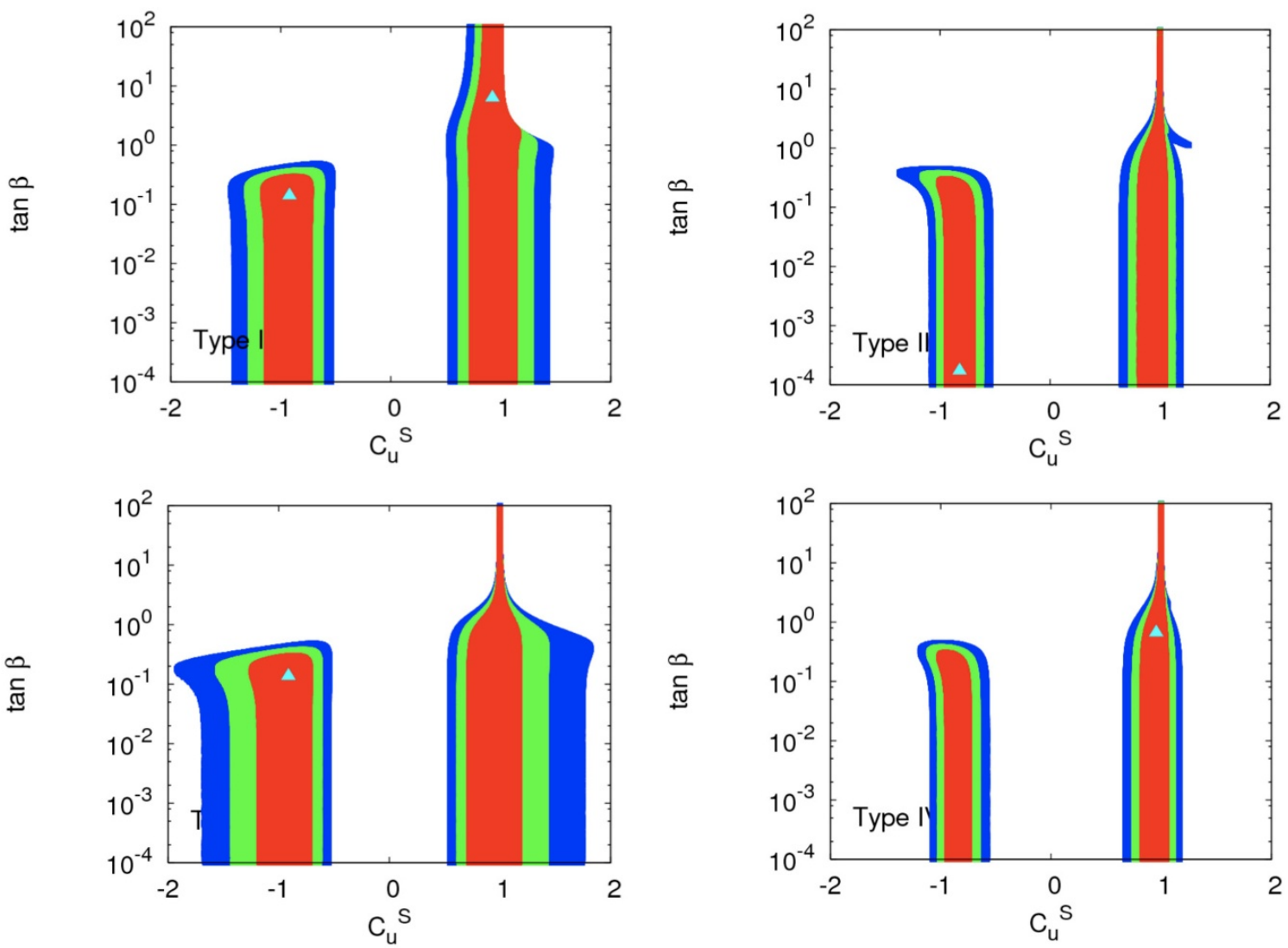

Figure 7. The same as figure 6 but in the plane of $C_{u}^{S}$ vs $\tan \beta$ for Type I-IV (CPC3). The description of the confidence regions is the same as figure 6 . 

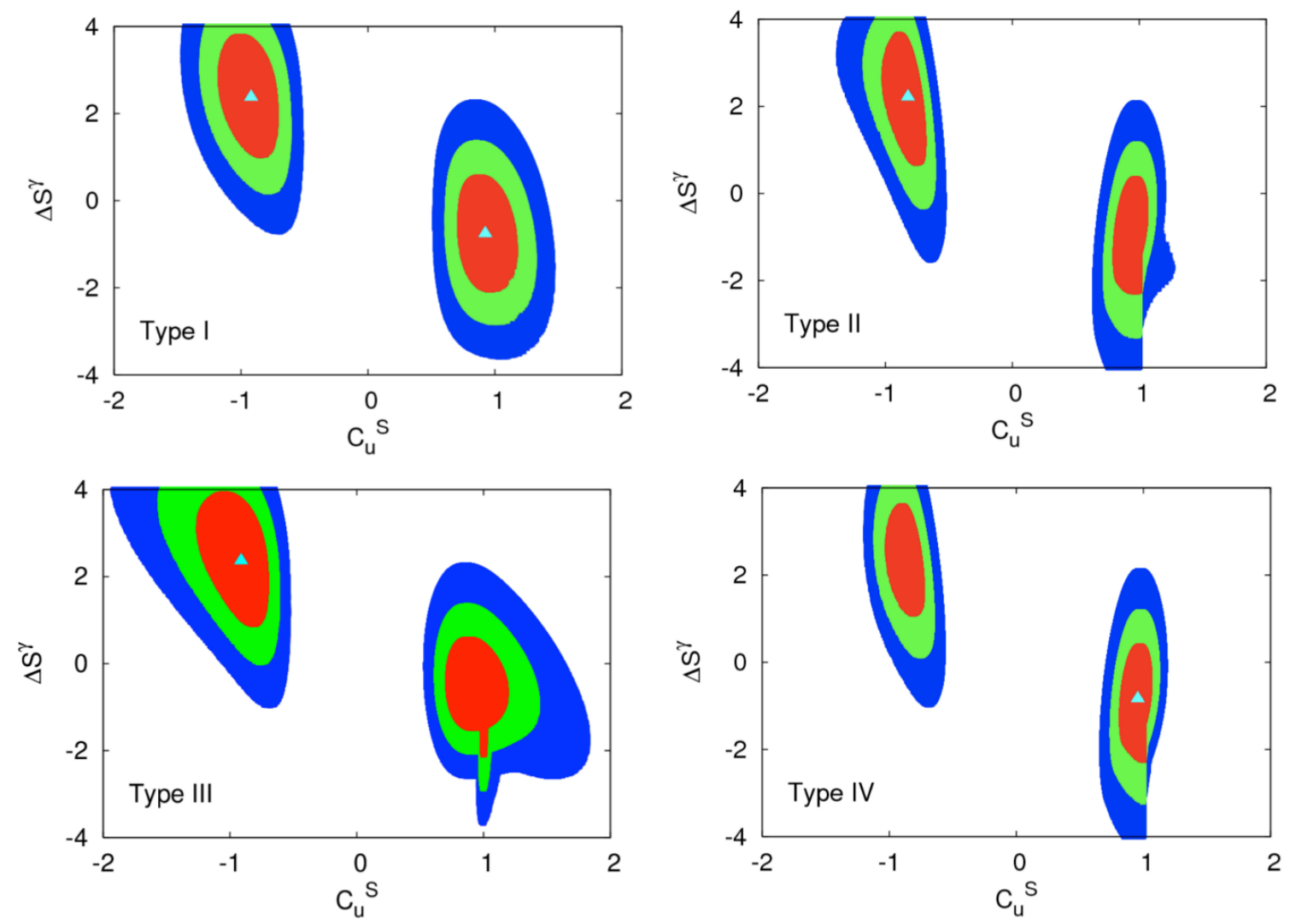

Figure 8. The same as figure 6 but in the plane of $C_{u}^{S}$ vs $\left(\Delta S^{\gamma}\right)^{H^{ \pm}}$for Type I-IV (CPC3). The description of the confidence regions is the same as figure 6 . 

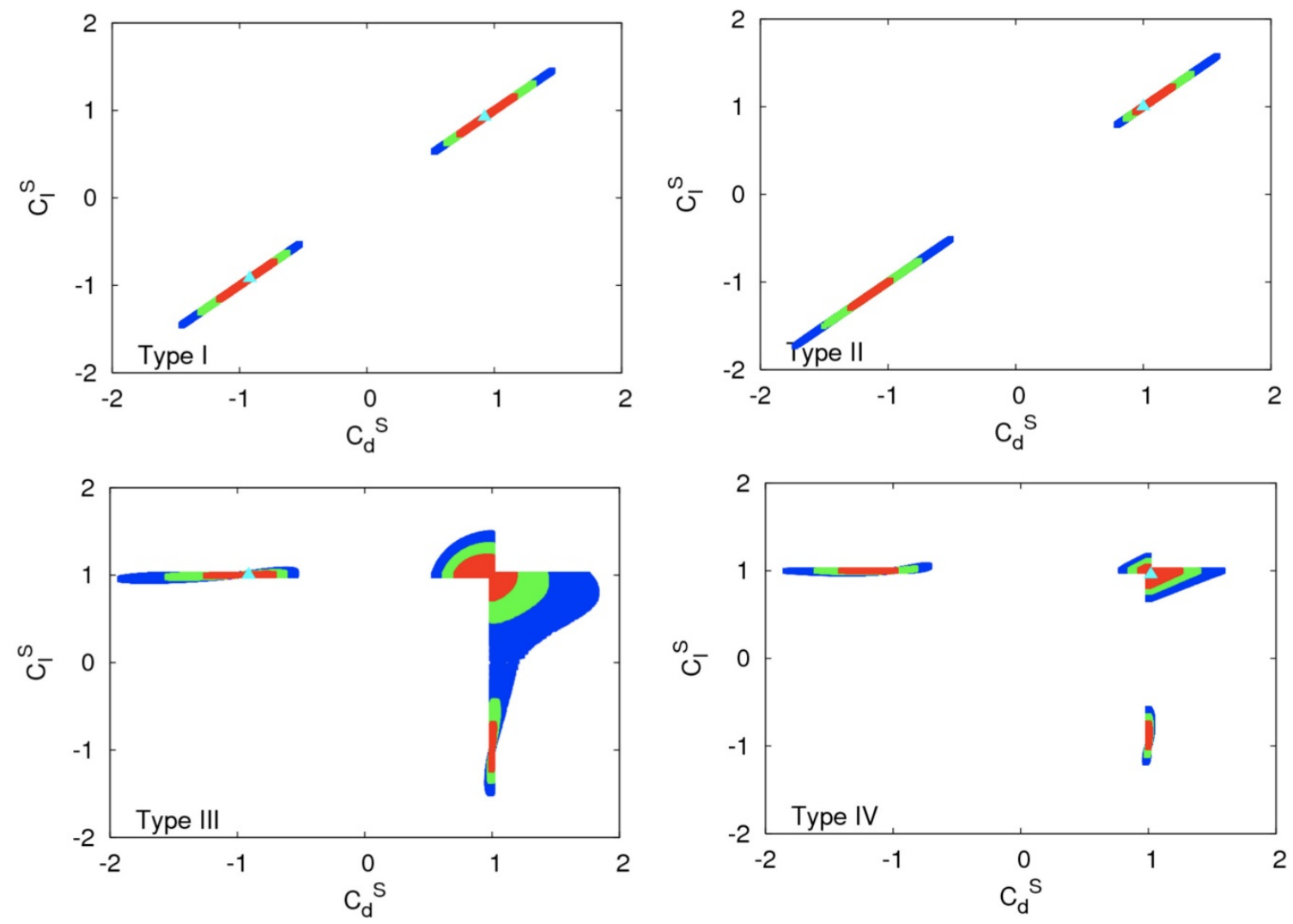

Figure 9. The same as figure 6 but in the plane of $C_{d}^{S}$ vs $C_{\ell}^{S}$ for Type I-IV (CPC3). The description of the confidence regions is the same as figure 6 . 

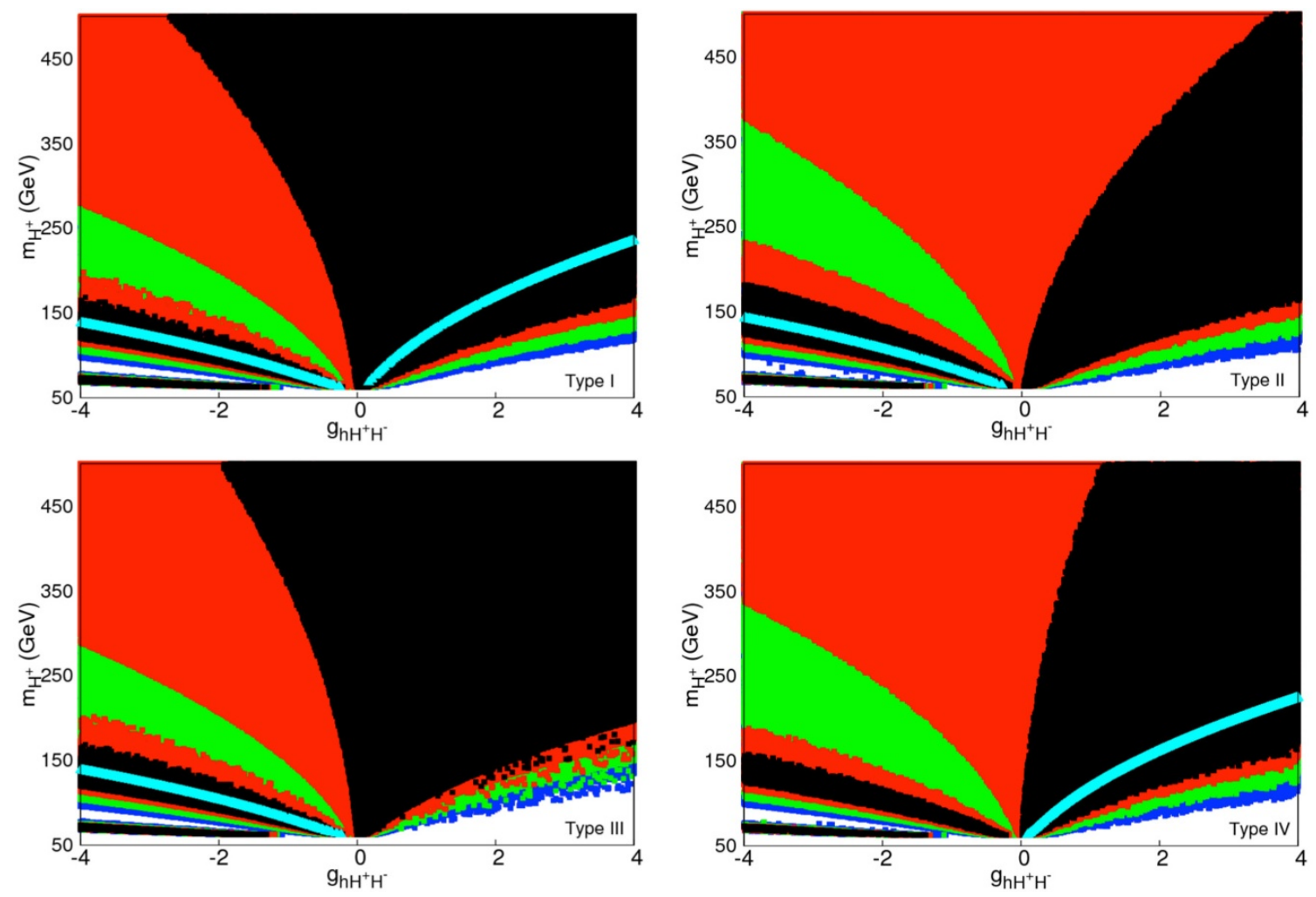

Figure 10. The same as figure 6 but we used $g_{h H^{+} H^{-}}$and $m_{H^{ \pm}}$in place of $\left(\Delta S^{\gamma}\right)^{H^{ \pm}}$(CPC3 case) for Type I-IV. The contour regions shown are for $\Delta \chi^{2} \leq 1.0$ (black), 2.3 (red), 5.99 (green), and 11.83 (blue) above the minimum, which correspond to confidence levels of $39.3 \%, 68.3 \%, 95 \%$, and $99.7 \%$, respectively. The best-fit points are denoted by a beam of cyan triangles. 


\subsection{CP violating fits}

In this subsection, we study the CP-violating case with a nonzero $C_{u}^{P}$ in addition to $C_{u}^{S}, C_{v}$ (or, equivalently, $\tan \beta$ ), and $\left(\Delta S^{\gamma}\right)^{H^{ \pm}}$. In our numerical study, we again find that $\tan \beta$ is bounded from above when $C_{u}^{S}$ deviates from its $\mathrm{SM}$ value 1 . So, as in the CP-conserving case, the precise and independent future measurements of $C_{u}^{S}$ and $\tan \beta$ can tell us the phenomenological viability of $2 \mathrm{HDM}$, thus providing some possible model discriminating power.

\subsubsection{CPV3}

In the CPV3 fit, we vary $C_{u}^{S}, C_{u}^{P}$, and $C_{v}$ (or equivalently $\log _{10} \tan \beta$ ). The other couplings $C_{d, l}^{S, P}$ are given by the relations shown in table 2 . The total $\chi^{2}, \chi^{2} /$ dof, $p$-value, and the best-fit values for $C_{u}^{S}, C_{u}^{P}$, and $C_{v}(\tan \beta)$ for the types I-IV 2 HDMs are shown in the lower half of table 3 . We show the contour plots for confidence-level regions as functions $C_{u}^{S}$ vs $C_{u}^{P}, C_{u}^{S}$ vs $C_{v}, C_{u}^{S}$ vs $\tan \beta, C_{d}^{S}$ vs $C_{d}^{P}$, and $C_{l}^{S}$ vs $C_{l}^{P}$ in figures $11-15$, respectively. We found that type II gives the smallest $\chi^{2}$ and the variation of total $\chi^{2}$ among the 4 types is within 1.2 , which is about 4 times larger compared to the CP-conserving case. Yet, such small $\chi^{2}$ differences cannot help us to preferentially select one of the types. The best $p$-value for type II is 0.578 , which is the largest among all the fits considered in this work, but it is still smaller than the $\mathrm{SM} p_{\mathrm{SM}}=0.65$.

The best-fit values for $C_{u}^{S}$ are all positive: 0.87 (I), 0.48 (II), 0.87 (III) and 0.81 (IV); while we have both the positive and negative best-fit values for $C_{u}^{P}: \pm 0.15$ (I), \pm 0.51 (II), \pm 0.11 (III) and \pm 0.34 (IV). Note that the largest (almost maximal) $\mathrm{CP}$ violation can occur in type II with $C_{u}^{S} \sim\left|C_{u}^{P}\right| \sim 0.5$. The best-fit values for $C_{v}$ are 0.99 (I) and 1 (II, III and IV), and those for $\tan \beta$ are 0.9 (I), 0.1 (II), and $\sim 10^{-4}$ (III and IV).

The CL regions in the $C_{u}^{S}$ and $C_{u}^{P}$ plane are shown in figure 11. A positive $C_{u}^{S}$ is in general preferred and it takes a value between 0.44 and 1.1 (I), -0.30 and 1.1 (II), 0.64 and 1.2 (III), and 0.26 and 1.1 (IV) at $68.3 \% \mathrm{CL}$. For $C_{u}^{P}$, the $68.3 \% \mathrm{CL}$ regions are between: -0.55 and +0.55 (I), -0.70 and +0.70 (II), -0.45 and +0.45 (III), and -0.73 and $+0.73(\mathrm{IV})$. We note that maximal CP violation with $C_{u}^{S} \sim\left|C_{u}^{P}\right|$ is possible even when $C_{v} \simeq 1$. This can be understood by considering the relation eq. (3.14), which takes on a form of

$$
C_{v}=1-\frac{1}{2} \beta^{2}\left[\left(C_{u}^{S}-1\right)^{2}+\left(C_{u}^{P}\right)^{2}\right]+\mathcal{O}\left(\beta^{3}\right)
$$

in the $\tan \beta=0$ limit. Taking an example of $C_{u}^{S}=C_{u}^{P}=1 / 2$, one may have

$$
O_{\phi_{2} i}=\beta / 2, \quad O_{a i}=-\beta / 2, \quad O_{\phi_{1} i}=1-\beta^{2} / 4, \quad C_{v}=1-\beta^{2} / 4
$$

up to $\mathcal{O}\left(\beta^{3}\right)$. Hence, although the $126-\mathrm{GeV}$ observed state is mostly CP-even dominated by the $\phi_{1}$ component, it can have maximally CP-violating couplings to the up-type quarks with $C_{u}^{S}=\left|C_{u}^{P}\right|=1 / 2$.

In figures 12 and 13, we show the CL regions in the $C_{u}^{S}$ vs $C_{v}$ and $C_{u}^{S}$ vs $\tan \beta$ planes, respectively. Compared to the CPC case, we observe that the two islands are now merged together, except for type III. We again find that $\tan \beta$ is bounded from 

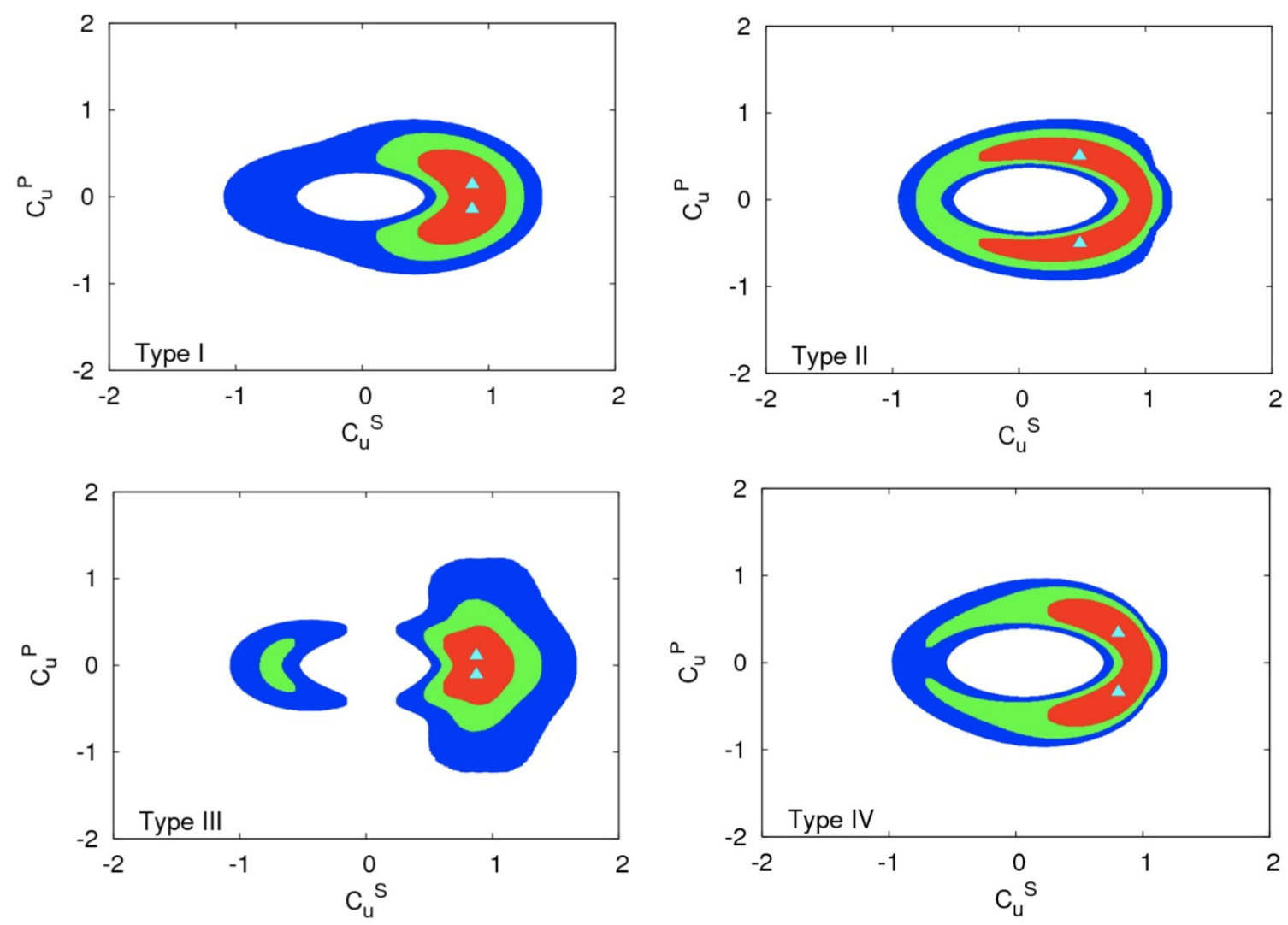

Figure 11. The confidence-level regions of the fit by varying $C_{u}^{S}, C_{u}^{P}$, and $\log _{10} \tan \beta$ (CPV3 case) in the plane of $C_{u}^{S}$ vs $C_{u}^{P}$ for Type I-IV. The contour regions shown are for $\Delta \chi^{2} \leq 2.3$ (red), 5.99 (green), and 11.83 (blue) above the minimum, which correspond to confidence levels of $68.3 \%$, $95 \%$, and $99.7 \%$, respectively. The best-fit points are denoted by the triangle.

above: $\tan \beta \lesssim 1$ (II), $\tan \beta \lesssim 3$ (III), and $\tan \beta \lesssim 2$ (IV). As in the CPC case, considerable deviation of $C_{u}^{S}$ from 1 for large $\tan \beta \gtrsim 10$ is not possible in the type II, III, IV models.

In figure 14, we show the Higgs couplings to the down-type quarks. The behavior can be understood by observing the relations $C_{d}^{S}=C_{u}^{S}$ and $C_{d}^{P}=-C_{u}^{P}$ (I and III) and $C_{d}^{S}= \pm\left\{1+t_{\beta}^{2}\left[1-\left(C_{u}^{S}\right)^{2}\right]-t_{\beta}^{4} / s_{\beta}^{2}\left(C_{u}^{P}\right)^{2}\right\}^{1 / 2}$ and $C_{d}^{P}=t_{\beta}^{2} C_{u}^{P}$ (II and IV), see table 2. Note that $\left|C_{d}^{P}\right| \lesssim 1$ at $99.7 \% \mathrm{CL}$. We observe large $\mathrm{CP}$ violation is possible in the Higgs couplings to the down-type quarks.

In figure 15, we show the Higgs couplings to the charged leptons. Now the couplings are given by $C_{l}^{S}=C_{u}^{S}$ and $C_{l}^{P}=-C_{u}^{P}$ (I and IV) and $C_{l}^{S}= \pm\left\{1+t_{\beta}^{2}[1-\right.$ $\left.\left.\left(C_{u}^{S}\right)^{2}\right]-t_{\beta}^{4} / s_{\beta}^{2}\left(C_{u}^{P}\right)^{2}\right\}^{1 / 2}$ and $C_{l}^{P}=t_{\beta}^{2} C_{u}^{P}$ (II and III). Again we note that $\left|C_{l}^{P}\right| \lesssim 1$ at $99.7 \% \mathrm{CL}$ and large $\mathrm{CP}$ violation is also possible in the Higgs couplings to the charged leptons.

Before we close this sub-subsection, we make a comment on the figures for the CL regions in the planes of $C_{d}^{S}$ vs $C_{d}^{P}$ and $C_{l}^{S}$ vs $C_{l}^{P}$. Unless $\left(C_{d, l}^{S}, C_{d, l}^{P}\right)=\left(C_{u}^{S},-C_{u}^{P}\right)$, the boundaries of the CL regions are somewhat fuzzy as shown in the frames for type II and IV 

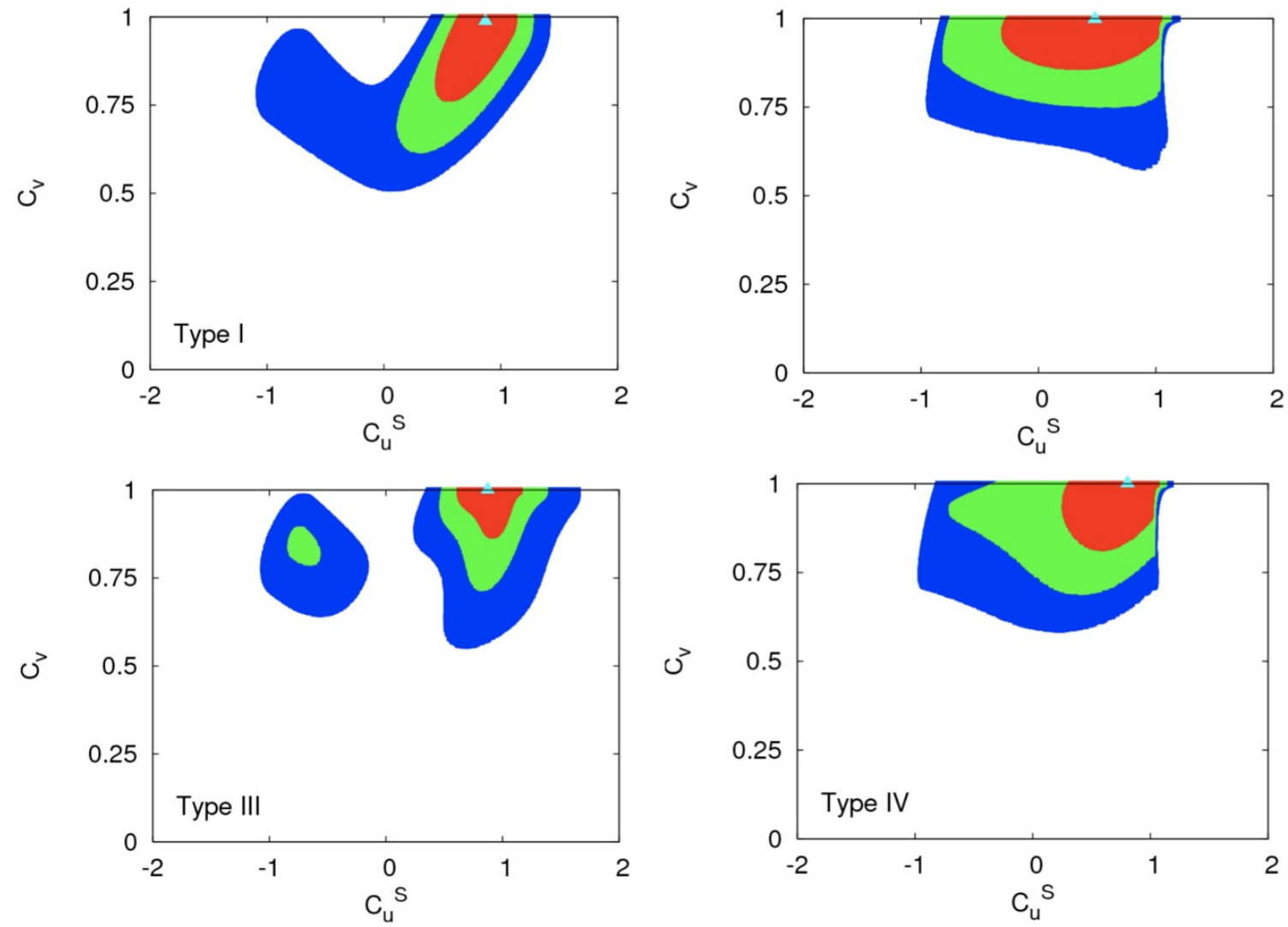

Figure 12. The same as in figure 11 but in the plane of $C_{u}^{S}$ vs $C_{v}$ for Type I-IV (CPV3). The description of the confidence regions is the same as figure 11.

of figure 14 and in those for type II and III of figure 15. We figure out that this is because one has $\left(C_{d, l}^{S}, C_{d, l}^{P}\right) \sim(1,0)$ in most of the parameters space due to the coupling relations shown in table 2. Furthermore, we have the fewer points on the negative side of $C_{d}^{S}$ or $C_{l}^{S}$. For the couplings $C_{d, l}^{S}$ to be negative, the negative sign needs to be chosen for $O_{\phi_{1} i}$ in eq. (3.13). But we note that the other positive sign is chosen mostly for $O_{\phi_{1} i}$ due to the choice of $C_{v}>0$ made in the analysis. Similar behavior happens in figure 20 and figure 21 .

\subsubsection{CPV4}

In the CPV4 fit, we vary $C_{u}^{S}, C_{u}^{P}, C_{v}$ (or $\log _{10} \tan \beta$ equivalently), and $\left(\Delta S^{\gamma}\right)^{H^{ \pm}}$. The other couplings $C_{d, l}^{S, P}$ are given by the relations shown in table 2 . The total $\chi^{2}, \chi^{2} /$ dof, $p$-value and the best-fit values for $C_{u}^{S}, C_{u}^{P}, C_{v}(\tan \beta)$, and $\left(\Delta S^{\gamma}\right)^{H^{ \pm}}$for the four types of 2 HDMs can be found in the lower half of table 3 . We show the contour plots for confidencelevel regions as functions $C_{u}^{S}$ vs $C_{u}^{P}, C_{u}^{S}$ vs $C_{v}, C_{u}^{S}$ vs $\tan \beta, C_{u}^{S}$ vs $\left(\Delta S^{\gamma}\right)^{H^{ \pm}}, C_{d}^{S}$ vs $C_{d}^{P}$, and $C_{l}^{S}$ vs $C_{l}^{P}$ in figures 16-21, respectively. We find that type II gives the smallest $\chi^{2}$ and its variation among the 4 types is within 0.57 , which is smaller than that of the CPV3 fits. The $p$-values of the CPV4 fits are also worse than the CPV3 fits. 

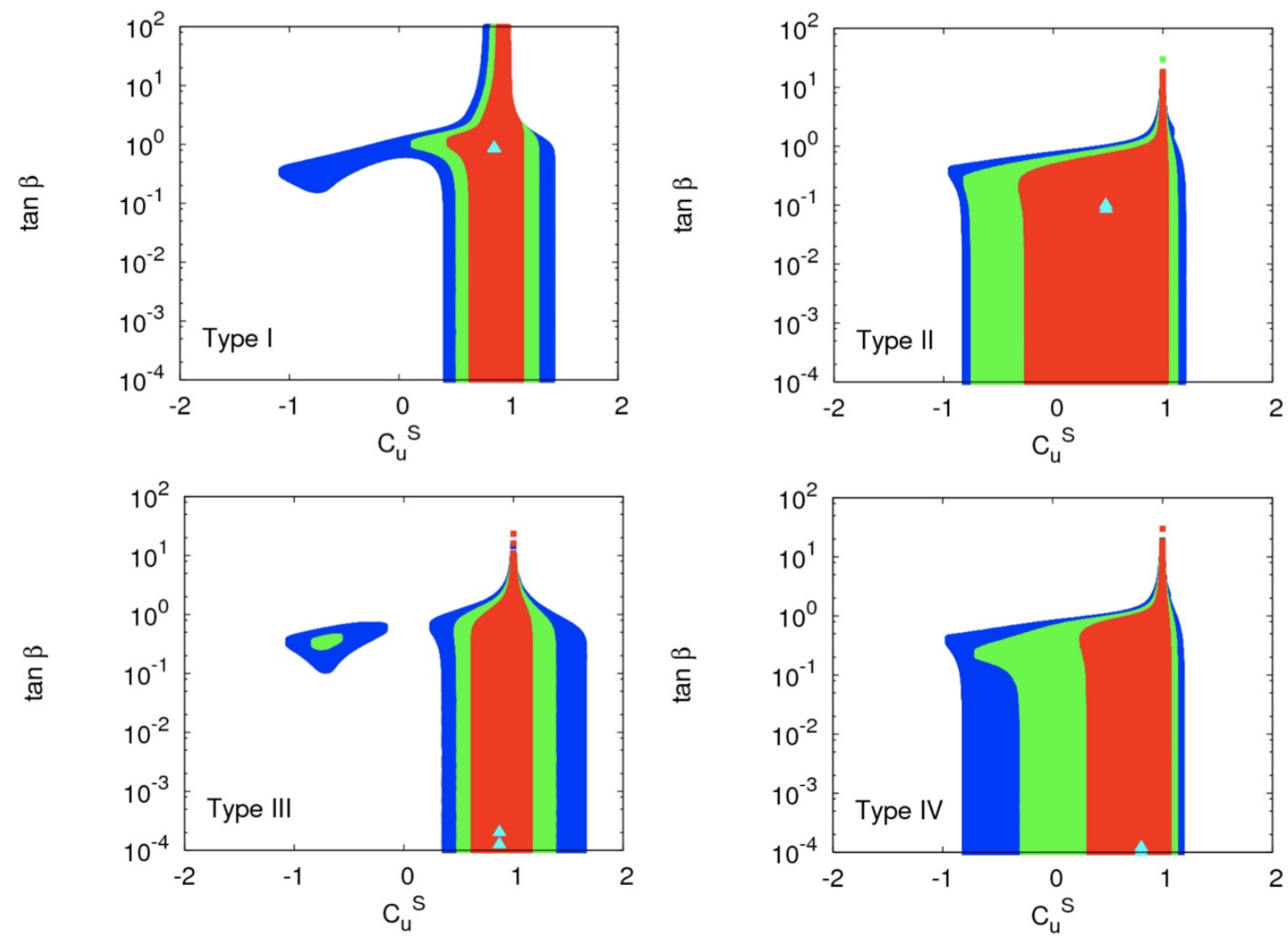

Figure 13. The same as in figure 11 but in the plane of $C_{u}^{S}$ vs $\tan \beta$ for Type I-IV (CPV3). The description of the confidence regions is the same as figure 11.

The best-fit values for $C_{u}^{S}$ are about \pm 0.92 (I), -0.05 (II), -0.91 (III) and 0.96 (IV), while those of $C_{u}^{P}$ are about 0 (I), \pm 0.57 (II), 0.03 (III), and -0.02 (IV). In type II, we note the best-fit value for $C_{u}^{S}$ is almost 0 and those of $C_{u}^{P}$ are very small except for type II. Therefore, in terms of the best-fit values the measure of $\mathrm{CP}$-violating effect $2 C_{u}^{S} C_{u}^{P} /\left[\left(C_{u}^{S}\right)^{2}+\left(C_{u}^{P}\right)^{2}\right]$ is not significant in all 4 types of 2 HDMs. Nevertheless, the CP violation could be significant taking account of the errors. For the Higgs couplings to the down-type quarks and charged leptons, we find that all the couplings $C_{d}^{P}$ and $C_{l}^{P}$ are almost vanishing: see table 4 . The best-fit values for $C_{v}$ are about 0.97 (I and III) and 1 (II and IV) and those for $\tan \beta$ are $\mathcal{O}(0.1)$, except for type I with positive $C_{u}^{S}$, where the best-fit value is 6.5. As will be shown below in the figures, variation of $\chi^{2}$ vs of $\tan \beta$ is small in a large region of parameter space. For $\left(\Delta S^{\gamma}\right)^{H^{ \pm}}$, the best-fit values are -0.78 and 2.4 (I), 1.0 (II), 2.4 (III), and -0.83 (IV). This also can be understood from eq. (4.5).

In figure 16, we show the CL regions in the $C_{u}^{S}$ and $C_{u}^{P}$ plane, and note that the positive and negative $C_{u}^{S}$ regions are providing equally good fits. The $68 \% \mathrm{CL}$ regions of $C_{u}^{S}$ are: $-1.1 \sim-0.5$ and $0.5 \sim 1.1$ (I), $-1 \sim 1$ (II), $-1.2 \sim-0.5$ and $0.6 \sim 1.2$ (III), and $-1 \sim-0.4$ and $0.3 \sim 1.1$ (IV). Also, $C_{u}^{P}$ varies between \pm 0.5 (I, III) and \pm 0.7 (II, IV) in the $68 \% \mathrm{CL}$ regions. Therefore, the maximal $\mathrm{CP}$ violation with $\left|C_{u}^{S}\right|=\left|C_{u}^{P}\right|$ is still possible. 

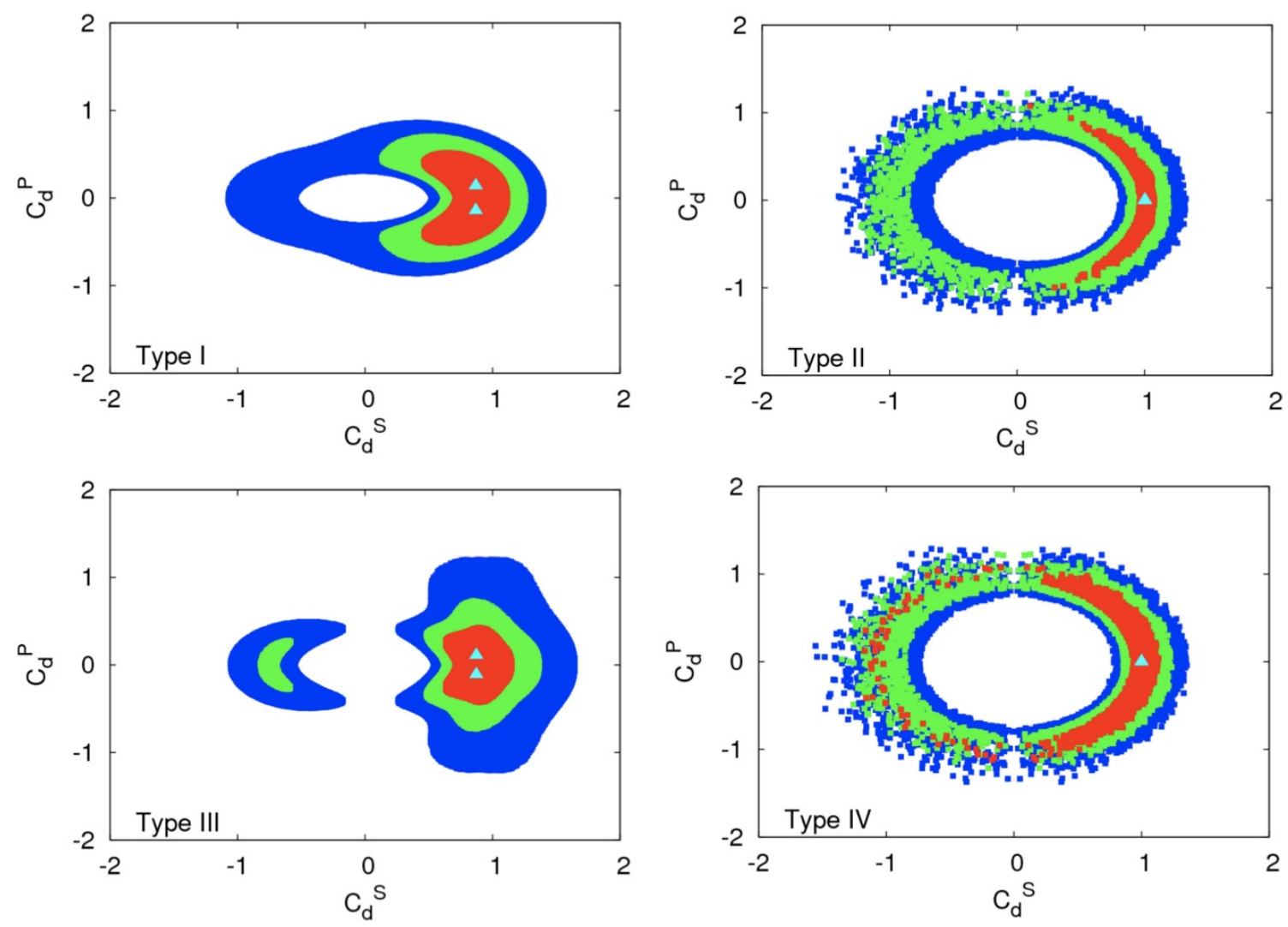

Figure 14. The same as in figure 11 but in the plane of $C_{d}^{S}$ vs $C_{d}^{P}$ for Type I-IV (CPV3). The description of the confidence regions is the same as figure 11.

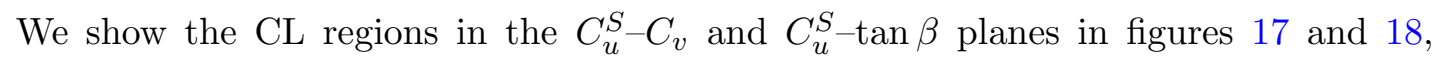
respectively. $C_{v} \gtrsim 0.8$ at $68 \% \mathrm{CL}$ and $\tan \beta$ are bounded from above for the type II, III, and IV, except for a narrow region around $C_{u}^{S}=1$. The CL regions in the plane of $C_{u}^{S}$ and $\left(\Delta S^{\gamma}\right)^{H^{ \pm}}$are shown in figure 19. Roughly speaking, $-2 \lesssim\left(\Delta S^{\gamma}\right)^{H^{ \pm}} \lesssim 3.5(68 \% \mathrm{CL})$. In figures 20 and 21, the Higgs couplings to the down-type quarks and charged leptons are shown.

Finally, the single parameter $\left(\Delta S^{\gamma}\right)^{H^{ \pm}}$can be interpreted in terms of the charged Higgs mass $M_{H^{ \pm}}$and the neutral Higgs coupling to the charged Higgses $g_{H_{i} H^{+} H^{-}}$, as in eq. (3.15). In figure 22, we show the CL regions in the plane of $M_{H^{ \pm}}$vs $g_{H_{i} H^{+} H^{-}}$. Compared to the CPC3 case, we have $\Delta \chi^{2} \leq 1$ in the wider range. 

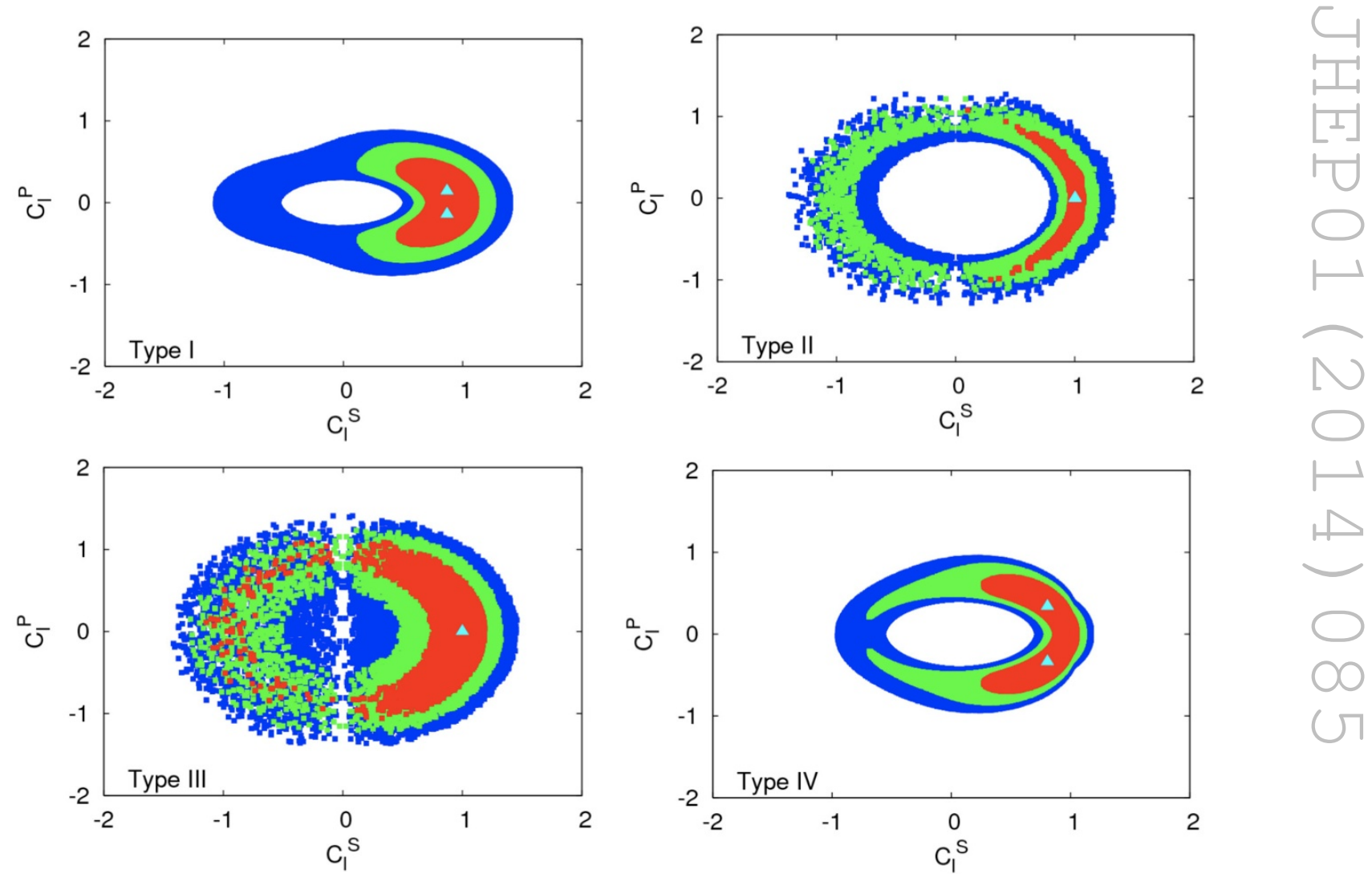

Figure 15. The same as in figure 11 but in the plane of $C_{l}^{S}$ vs $C_{l}^{P}$ for Type I-IV (CPV3). The description of the confidence regions is the same as figure 11. 

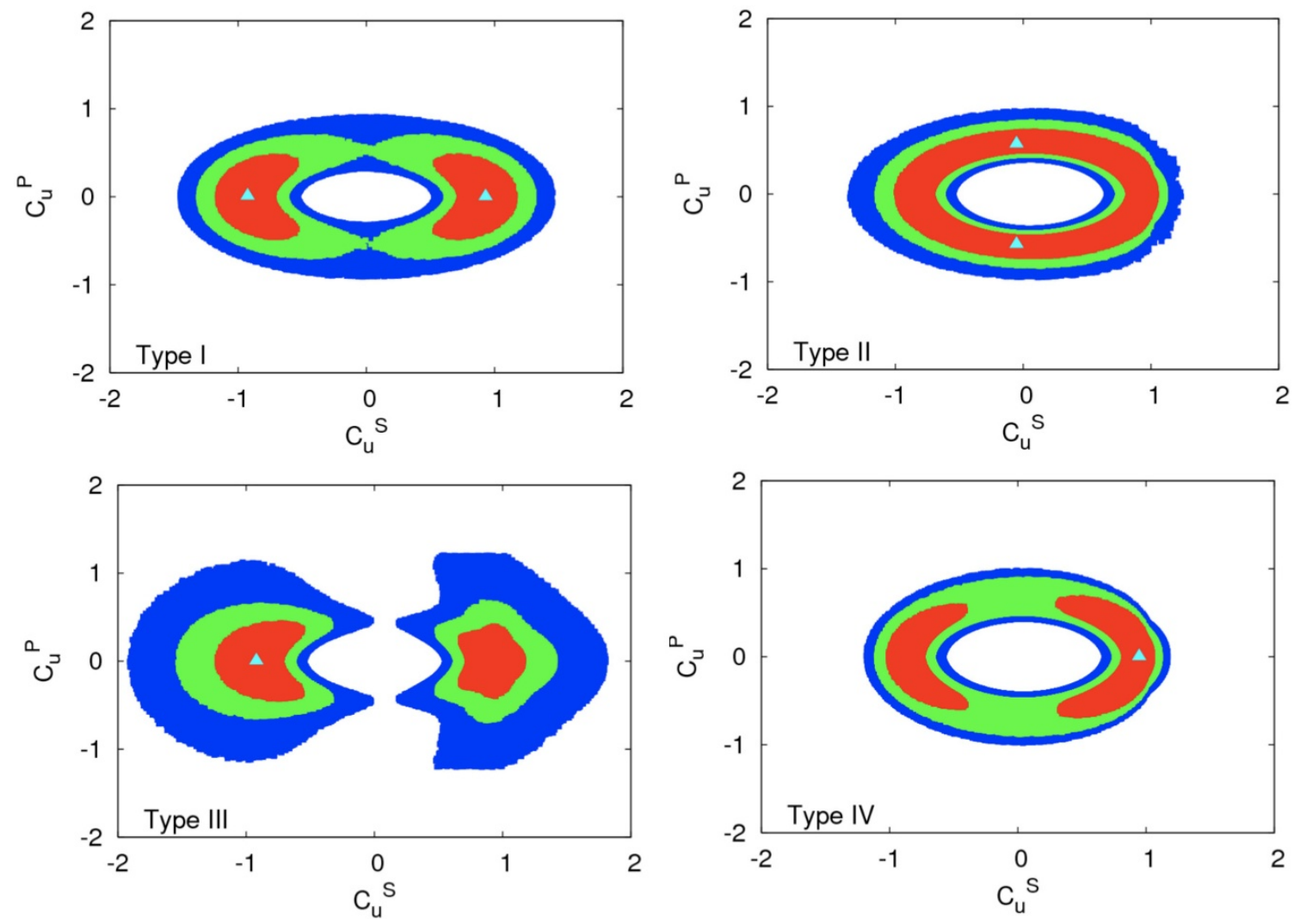

Figure 16. The confidence-level regions of the fit by varying $C_{u}^{S}, C_{u}^{P}, \log _{10} \tan \beta$, and $\left(\Delta S^{\gamma}\right)^{H^{ \pm}}$ (CPV4 case) in the plane of $C_{u}^{S}$ vs $C_{u}^{P}$ for Type I-IV. The contour regions shown are for $\Delta \chi^{2} \leq 2.3$ (red), 5.99 (green), and 11.83 (blue) above the minimum, which correspond to confidence levels of $68.3 \%, 95 \%$, and $99.7 \%$, respectively. The best-fit points are denoted by the triangle. 

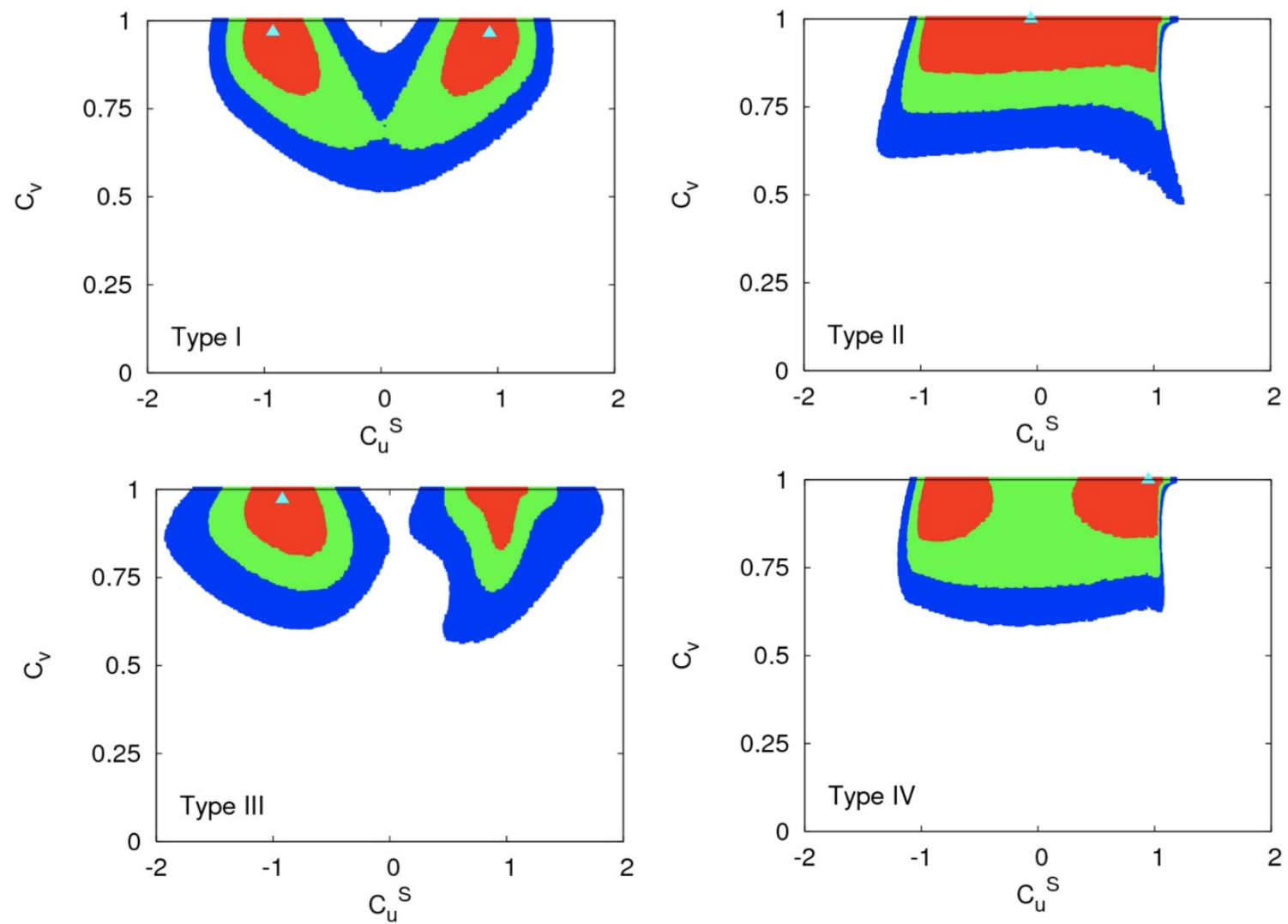

Figure 17. The same as figure 16 but in the plane of $C_{u}^{S}$ vs $C_{v}$ for Type I-IV (CPV4). The description of the confidence regions is the same as figure 16 . 

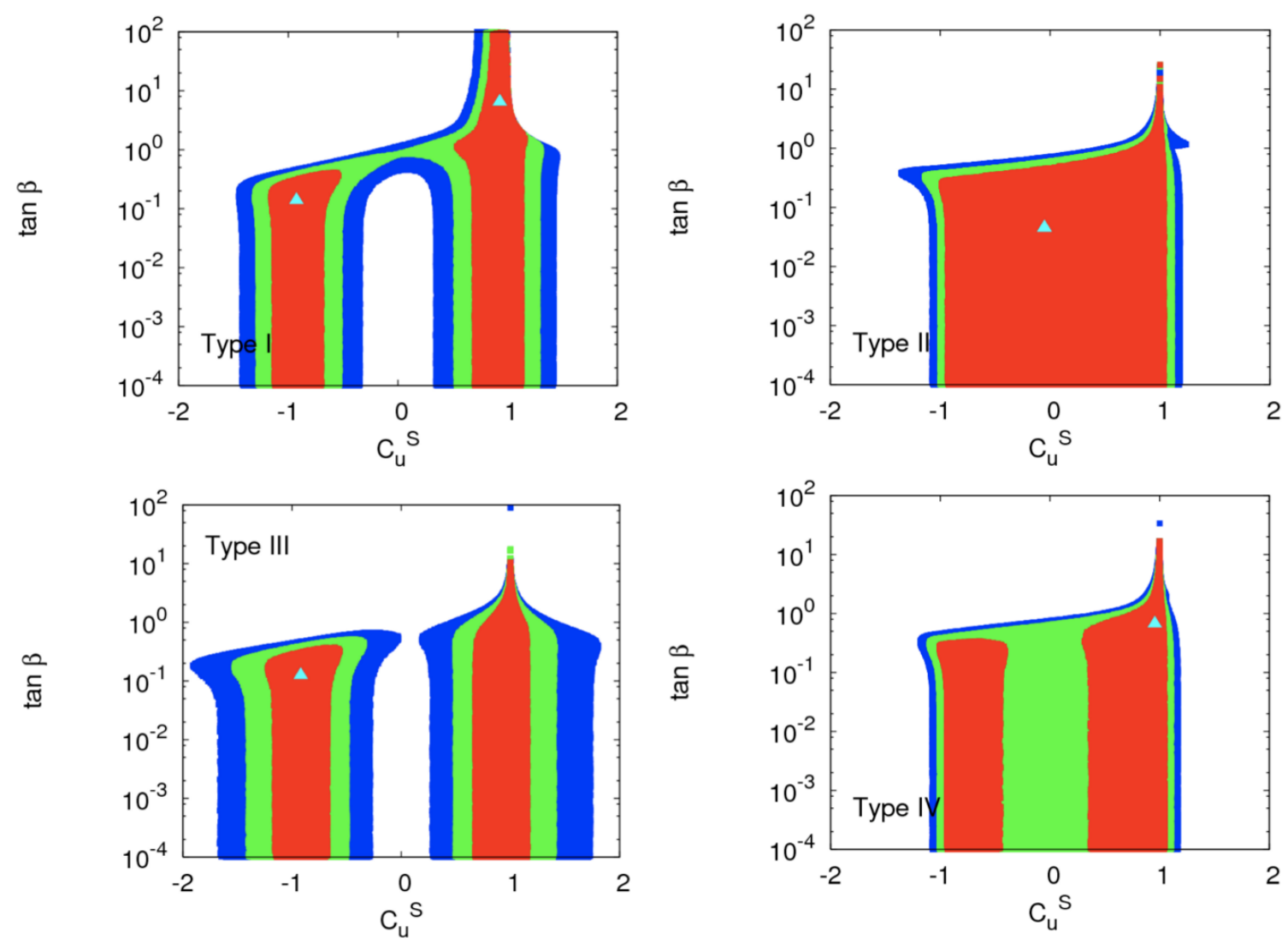

Figure 18. The same as figure 16 but in the plane of $C_{u}^{S}$ vs $\tan \beta$ for Type I-IV (CPV4 case). The description of the confidence regions is the same as figure 16 . 

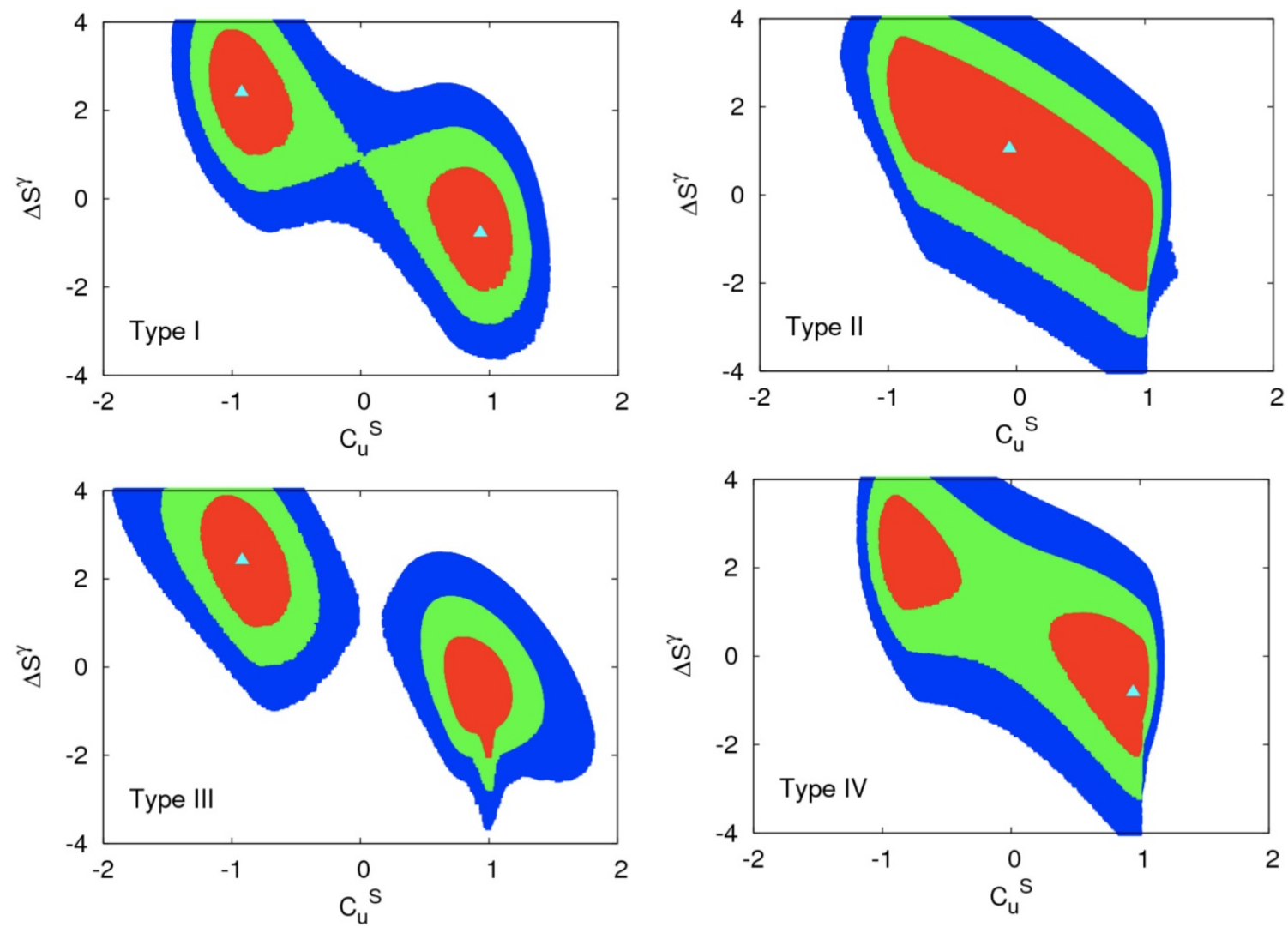

Figure 19. The same as figure 16 but in the plane of $C_{u}^{S}$ vs $\left(\Delta S^{\gamma}\right)^{H^{ \pm}}$for Type I-IV (CPV4). The description of the confidence regions is the same as figure 16 . 

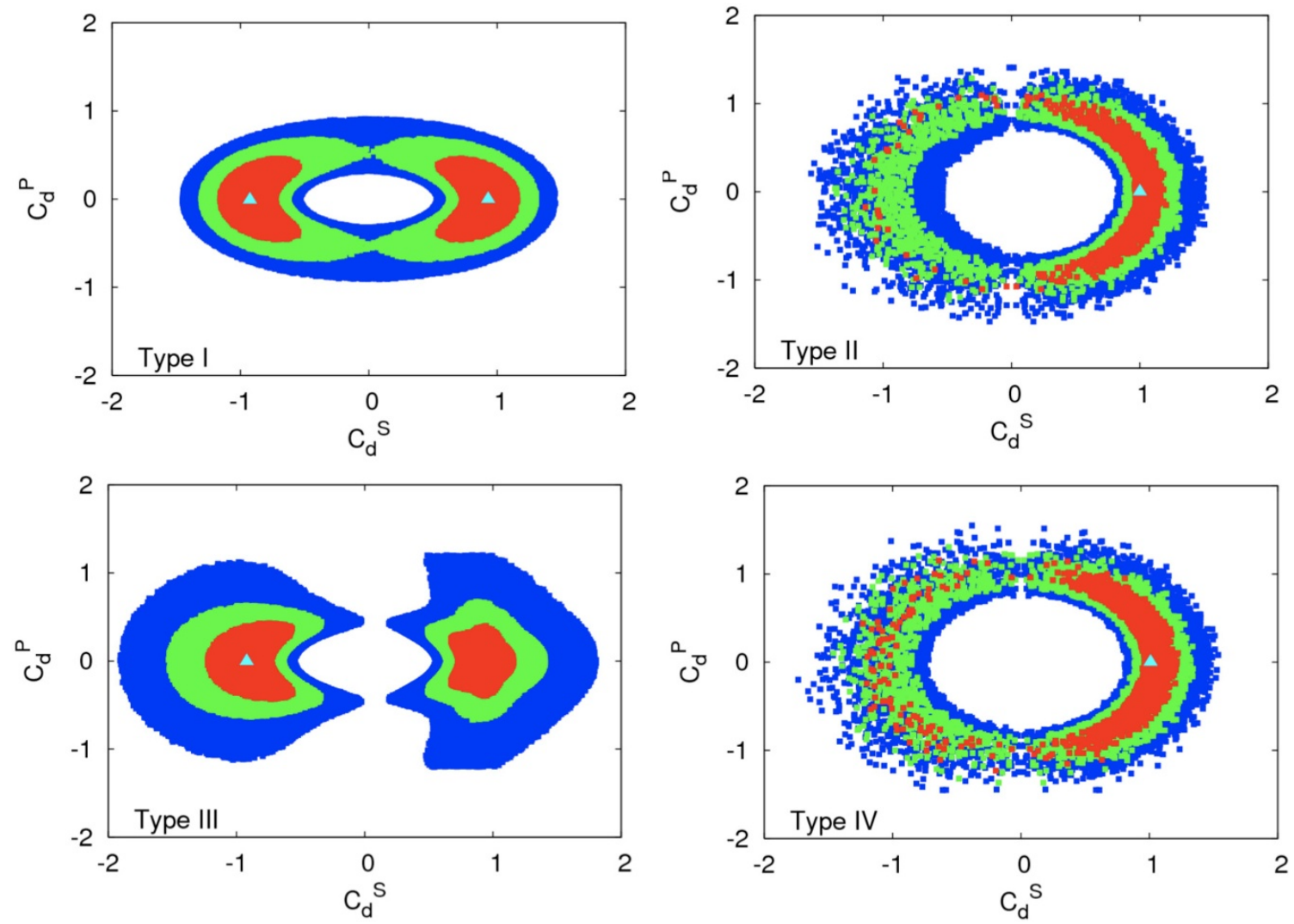

Figure 20. The same as figure 16 but in the plane of $C_{d}^{S}$ vs $C_{d}^{P}$ for Type I-IV (CPV4). The description of the confidence regions is the same as figure 16 . 

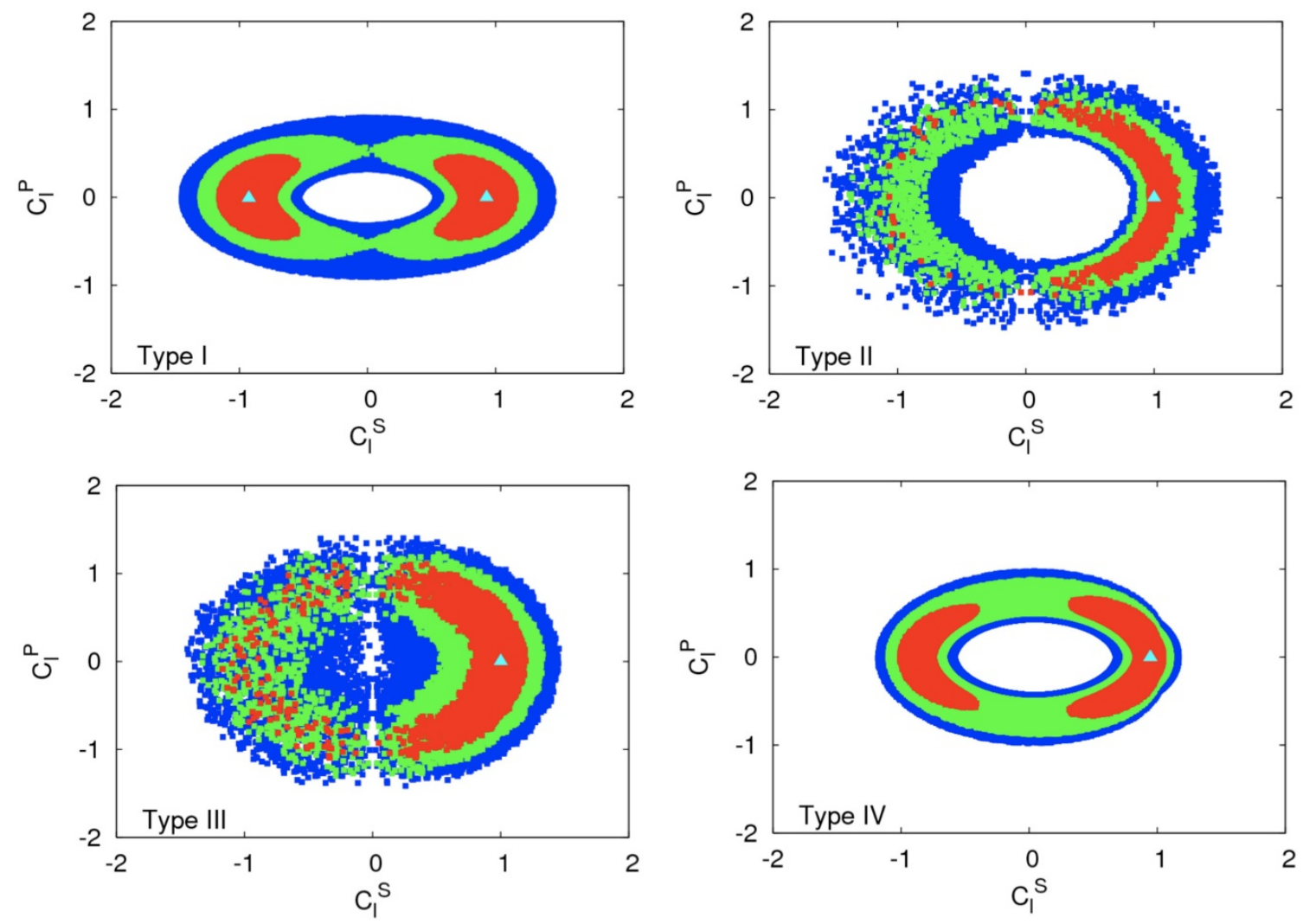

Figure 21. The same as figure 16 but in the plane of $C_{\ell}^{S}$ vs $C_{\ell}^{P}$ for Type I-IV (CPV4). The description of the confidence regions is the same as figure 16 . 

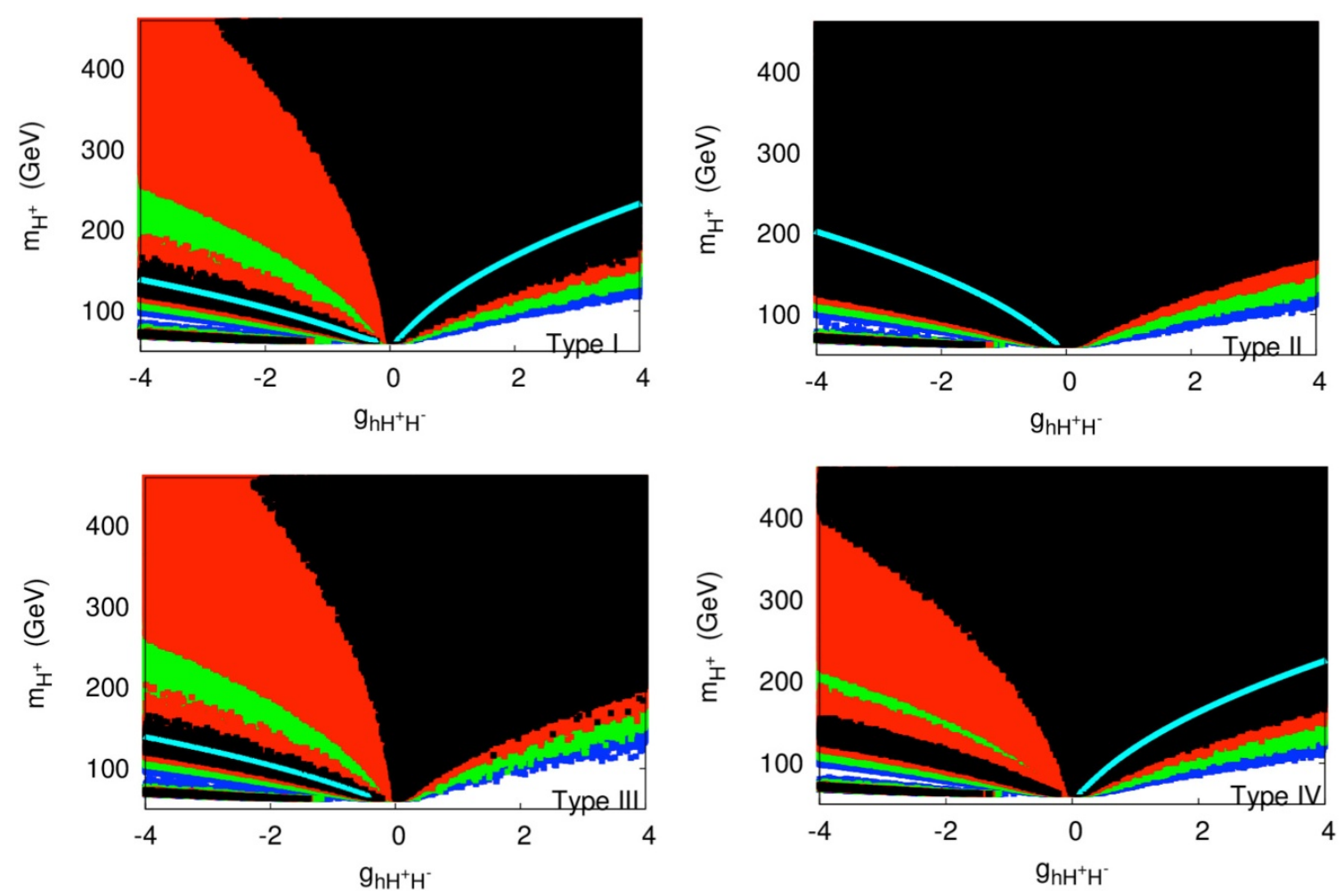

Figure 22. The same as figure 16 but we used $g_{h H^{+} H^{-}}$and $m_{H^{ \pm}}$in place of $\left(\Delta S^{\gamma}\right)^{H^{ \pm}}$for Type IIV (CPV4). The contour regions shown are for $\Delta \chi^{2} \leq 1.0$ (black), 2.3 (red), 5.99 (green), and 11.83 (blue) above the minimum, which correspond to confidence levels of $39.3 \%, 68.3 \%, 95 \%$, and $99.7 \%$, respectively. The best-fit points are denoted by a beam of cyan triangles. 


\section{Discussion}

In this work, we have applied our previous model-independent approach [6], which analyzes all the observed Higgs boson signal strengths and fits to all the Higgs boson couplings, to the 2HDMs. In 2HDMs, the Higgs couplings to up-type and down-type quarks, and charged leptons are related by a set of relations shown in table 2 . We have shown that the whole analysis can be performed with at most 3 independent parameters: $C_{u}^{S}, C_{v}$ (or $\tan \beta)$, and $\left(\Delta S^{\gamma}\right)^{H^{ \pm}}$for CP-conserving scenarios, and only one more parameter $C_{u}^{P}$ for the CP-violating scenarios. A number of relationships among the couplings of the upand down-type quarks and charged leptons have been derived such that we need only $C_{u}^{S}$ and $C_{u}^{P}$.

A set of discrete symmetries are often imposed in literature in order to eliminate flavor-changing neutral currents, denoted by the parameters $\eta_{1,2}^{d, l}$, which take up the values either 0 or 1 . The four combinations of $\left(\eta_{1}^{d}, \eta_{1}^{l}\right)=(0,0),(1,1),(0,1),(1,0)$ correspond to type I, II, III, and IV, respectively. We have demonstrated that the current Higgs boson data have no preference for any of the four types of $2 \mathrm{HDMs}$, because statistically the $\chi^{2}$ difference among type I-IV is only 0.3 for CPC cases and 1.2 for CPV cases: see table 3. We also relaxed the discrete symmetries to allow continuous values for $\eta_{1,2}^{d, l}$ subject to normalization $\left(\eta_{1}^{d, l}\right)^{2}+\left(\eta_{2}^{d, l}\right)^{2}=1$, and we found that in the whole plane of $0 \leq \eta_{1}^{d}, \eta_{1}^{l} \leq 1$ the $\chi^{2}$ differences among the best-fits are all within $\chi^{2}<1$. . It is one of the main findings in this work - no particular preference among type I to IV as long as the current Higgs boson data are concerned.

The Higgs data used are almost the final set out of the $7 \mathrm{TeV}$ and $8 \mathrm{TeV}$ runs at the LHC. Further improvement to the fits will only be possible when more data are pouring in the next run of 2015. So far, the data have pointed to the SM Higgs boson with a large $p$-value, while all other extensions to the SM, such as the 2HDMs studied in this work or more model-independently in ref. [6], provide fits with smaller $p$-values than the SM. It means that the SM Higgs boson is currently the best explanation to all the Higgs boson data.

We offer a few more comments before we conclude.

1. The up-type and down-type (charged lepton) Yukawa couplings are related by quark masses and $\tan \beta$. Therefore, one set of parameters $C_{u}^{S}, C_{u}^{P}$, and $\tan \beta$ is sufficient to define all the fermionic couplings.

2. When we relax the discrete symmetries by varying $\eta_{1}^{d}$ and $\eta_{1}^{l}$, we found the best-fit values for them are neither 0 nor 1 . However, the $\chi^{2}$ differences in the whole plane of $\eta_{1}^{d}$ vs $\eta_{1}^{l}$ are too small to claim any preference statistically.

3. The charged Higgs boson contributes to the one-loop vertex $H \gamma \gamma$. In the studies, we first treated $\left(\Delta S^{\gamma}\right)^{H^{ \pm}}$as an independent parameter. Then we broke it down into the charged Higgs mass $M_{H^{ \pm}}$and the coupling $g_{H_{i} H^{+} H^{-}}$. When the $b \rightarrow s \gamma$ constraint (roughly $M_{H^{ \pm}}>300 \mathrm{GeV}$ ) is taken into account, positive $g_{H_{i} H^{+} H^{-}}$is preferred. 
4. The Higgs coupling to gauge bosons $C_{v}$ is constrained to be very close to 1 . It means that the observed Higgs boson is entirely responsible for breaking the electroweak symmetry.

5. Future precision measurements of $C_{u}^{S}$ and $\tan \beta$ can provide us with the discriminating power among various types of $2 \mathrm{HDMs}$ especially when $C_{u}^{S}$ deviates from its SM value 1.

6. The parameters $C_{u}^{S}$ and $C_{u}^{P}$ are constrained in the form of some ellipses. The current Higgs observables are not sensitive to $\mathrm{CP}$-violating effects, and so only combinations of scalar and pseudoscalar Yukawa couplings are constrained, as shown in figures 11 and 16 .

7. Among the $2 \mathrm{HDM}$ fits considered in this work, the type-II CP-violating case with $\left(\Delta S^{\gamma}\right)^{H^{ \pm}}=0$ (the CPV3 type-II fit) gives the best fit with $\chi^{2}=17.17$ and $p$ value $=0.578$ when $C_{u}^{S} \sim\left|C_{u}^{P}\right|=1 / 2$.

\section{Acknowledgments}

This work was supported the National Science Council of Taiwan under Grants No. 992112-M-007-005-MY3 and 102-2112-M-007-015-MY3. J.S.L. was supported by the National Research Foundation of Korea (NRF) grant (No. 2013R1A2A2A01015406). This study was also financially supported by Chonnam National University, 2012.

Open Access. This article is distributed under the terms of the Creative Commons Attribution License (CC-BY 4.0), which permits any use, distribution and reproduction in any medium, provided the original author(s) and source are credited.

\section{References}

[1] ATLAS collaboration, Observation of a new particle in the search for the standard model Higgs boson with the ATLAS detector at the LHC, Phys. Lett. B 716 (2012) 1 [arXiv: 1207.7214] [INSPIRE].

[2] CMS collaboration, Observation of a new boson at a mass of $125 \mathrm{GeV}$ with the CMS experiment at the LHC, Phys. Lett. B 716 (2012) 30 [arXiv:1207.7235] [INSPIRE].

[3] P.W. Higgs, Broken symmetries and the masses of gauge bosons, Phys. Rev. Lett. 13 (1964) 508 [INSPIRE].

[4] F. Englert and R. Brout, Broken symmetry and the mass of gauge vector mesons, Phys. Rev. Lett. 13 (1964) 321 [INSPIRE].

[5] G.S. Guralnik, C.R. Hagen and T.W.B. Kibble, Global conservation laws and massless particles, Phys. Rev. Lett. 13 (1964) 585 [InSPIRE].

[6] K. Cheung, J.S. Lee and P.-Y. Tseng, Higgs precision (Higgcision) era begins, JHEP 05 (2013) 134 [arXiv: 1302.3794] [INSPIRE]. 
[7] ATLAS collaboration, Measurements of the properties of the Higgs-like boson in the two photon decay channel with the ATLAS detector using $25 \mathrm{fb}^{-1}$ of proton-proton collision data, ATLAS-CONF-2013-012 (2013).

[8] ATLAS collaboration, Combined coupling measurements of the Higgs-like boson with the ATLAS detector using up to $25 \mathrm{fb}^{-1}$ of proton-proton collision data, ATLAS-CONF-2013-034 (2013).

[9] CMS collaboration, Updated measurements of the Higgs boson at $125 \mathrm{GeV}$ in the two photon decay channel, CMS-PAS-HIG-13-001 (2013).

[10] CMS collaboration, Properties of the Higgs-like boson in the decay $H \rightarrow Z Z \rightarrow 4 l$ in $p p$ collisions at $\sqrt{s}=7$ and $8 \mathrm{TeV}$, CMS-PAS-HIG-13-002 (2013).

[11] CMS collaboration, Update on the search for the standard model Higgs boson in pp collisions at the LHC decaying to $W^{+} W^{-}$in the fully leptonic final state, CMS-PAS-HIG-13-003 (2013).

[12] CMS collaboration, Search for the standard-model Higgs boson decaying to tau pairs in proton-proton collisions at $\sqrt{s}=7$ and $8 \mathrm{TeV}$, CMS-PAS-HIG-13-004 (2013).

[13] A. Juste, Standard model Higgs boson searches at the Tevatron, talk given at Hadron Collider Physics Symposium 2012, Kyoto Japan, 15 Nov 2012, http://kds.kek.jp/conferenceDisplay.py?confId $=9237$.

[14] Y. Enari, $H \rightarrow b \bar{b}$ from Tevatron, talk given at Hadron Collider Physics Symposium 2012, Kyoto Japan, 15 Nov 2012, http://kds.kek.jp/conferenceDisplay.py?confId=10808.

[15] D. Carmi, A. Falkowski, E. Kuflik and T. Volansky, Interpreting LHC Higgs results from natural new physics perspective, JHEP 07 (2012) 136 [arXiv:1202.3144] [INSPIRE].

[16] A. Azatov, R. Contino and J. Galloway, Model-independent bounds on a light Higgs, JHEP 04 (2012) 127 [Erratum ibid. 04 (2013) 140] [arXiv:1202.3415] [INSPIRE].

[17] J.R. Espinosa, C. Grojean, M. Muhlleitner and M. Trott, Fingerprinting Higgs suspects at the LHC, JHEP 05 (2012) 097 [arXiv: 1202.3697] [InSPIRE].

[18] M. Klute, R. Lafaye, T. Plehn, M. Rauch and D. Zerwas, Measuring Higgs couplings from LHC data, Phys. Rev. Lett. 109 (2012) 101801 [arXiv:1205.2699] [INSPIRE].

[19] D. Carmi, A. Falkowski, E. Kuflik and T. Volansky, Interpreting the Higgs, Frascati Phys. Ser. 57 (2013) 315 [arXiv:1206.4201] [INSPIRE].

[20] I. Low, J. Lykken and G. Shaughnessy, Have we observed the Higgs (imposter)?, Phys. Rev. D 86 (2012) 093012 [arXiv:1207.1093] [InSPIRE].

[21] P.P. Giardino, K. Kannike, M. Raidal and A. Strumia, Is the resonance at $125 \mathrm{GeV}$ the Higgs boson?, Phys. Lett. B 718 (2012) 469 [arXiv:1207.1347] [InSPIRE].

[22] J. Ellis and T. You, Global analysis of the Higgs candidate with mass $\sim 125 \mathrm{GeV}$, JHEP 09 (2012) 123 [arXiv: 1207.1693] [INSPIRE].

[23] J.R. Espinosa, C. Grojean, M. Muhlleitner and M. Trott, First glimpses at Higgs' face, JHEP 12 (2012) 045 [arXiv:1207.1717] [INSPIRE].

[24] D. Carmi, A. Falkowski, E. Kuflik, T. Volansky and J. Zupan, Higgs after the discovery: a status report, JHEP 10 (2012) 196 [arXiv:1207.1718] [INSPIRE].

[25] S. Banerjee, S. Mukhopadhyay and B. Mukhopadhyaya, New Higgs interactions and recent data from the LHC and the Tevatron, JHEP 10 (2012) 062 [arXiv:1207.3588] [INSPIRE]. 
[26] F. Bonnet, T. Ota, M. Rauch and W. Winter, Interpretation of precision tests in the Higgs sector in terms of physics beyond the standard model, Phys. Rev. D 86 (2012) 093014 [arXiv: 1207.4599] [INSPIRE].

[27] T. Plehn and M. Rauch, Higgs couplings after the discovery, Europhys. Lett. 100 (2012) 11002 [arXiv:1207.6108] [INSPIRE].

[28] A. Djouadi, Precision Higgs coupling measurements at the LHC through ratios of production cross sections, Eur. Phys. J. C 73 (2013) 2498 [arXiv:1208.3436] [InSPIRE].

[29] B.A. Dobrescu and J.D. Lykken, Coupling spans of the Higgs-like boson, JHEP 02 (2013) 073 [arXiv: 1210.3342] [InSPIRE].

[30] G. Cacciapaglia, A. Deandrea, G.D. La Rochelle and J.-B. Flament, Higgs couplings beyond the standard model, JHEP 03 (2013) 029 [arXiv: 1210.8120] [INSPIRE].

[31] G. Bélanger, B. Dumont, U. Ellwanger, J.F. Gunion and S. Kraml, Higgs couplings at the end of 2012, JHEP 02 (2013) 053 [arXiv: 1212.5244] [INSPIRE].

[32] G. Moreau, Constraining extra-fermion(s) from the Higgs boson data, Phys. Rev. D 87 (2013) 015027 [arXiv: 1210.3977] [INSPIRE].

[33] P.P. Giardino, K. Kannike, I. Masina, M. Raidal and A. Strumia, The universal Higgs fit, arXiv:1303.3570 [INSPIRE].

[34] P. Bechtle, S. Heinemeyer, O. Stål, T. Stefaniak and G. Weiglein, HiggsSignals: confronting arbitrary Higgs sectors with measurements at the Tevatron and the LHC, arXiv:1305.1933 [INSPIRE].

[35] A. Djouadi and G. Moreau, The couplings of the Higgs boson and its CP properties from fits of the signal strengths and their ratios at the $7+8 \mathrm{TeV} L H C$, arXiv:1303.6591 [INSPIRE].

[36] A. Falkowski, F. Riva and A. Urbano, Higgs at last, JHEP 11 (2013) 111 [arXiv:1303.1812] [INSPIRE].

[37] T. Corbett, O.J.P. Eboli, J. Gonzalez-Fraile and M.C. Gonzalez-Garcia, Constraining anomalous Higgs interactions, Phys. Rev. D 86 (2012) 075013 [arXiv:1207.1344] [INSPIRE].

[38] T. Corbett, O.J.P. Eboli, J. Gonzalez-Fraile and M.C. Gonzalez-Garcia, Robust determination of the Higgs couplings: power to the data, Phys. Rev. D 87 (2013) 015022 [arXiv:1211.4580] [INSPIRE].

[39] E. Masso and V. Sanz, Limits on anomalous couplings of the Higgs to electroweak gauge bosons from LEP and LHC, Phys. Rev. D 87 (2013) 033001 [arXiv:1211.1320] [INSPIRE].

[40] E. Boos, V. Bunichev, M. Dubinin and Y. Kurihara, Higgs boson signal at complete tree level in the SM extension by dimension-six operators, arXiv:1309.5410 [INSPIRE].

[41] S. Banerjee, S. Mukhopadhyay and B. Mukhopadhyaya, Higher dimensional operators and LHC Higgs data: the role of modified kinematics, arXiv:1308.4860 [INSPIRE].

[42] J. Cao, P. Wan, J.M. Yang and J. Zhu, The SM extension with color-octet scalars: diphoton enhancement and global fit of LHC Higgs data, JHEP 08 (2013) 009 [arXiv:1303.2426] [INSPIRE].

[43] H.S. Cheon and S.K. Kang, Constraining parameter space in type-II two-Higgs doublet model in light of a $126 \mathrm{GeV}$ Higgs boson, JHEP 09 (2013) 085 [arXiv:1207.1083] [INSPIRE].

[44] N. Craig and S. Thomas, Exclusive signals of an extended Higgs sector, JHEP 11 (2012) 083 [arXiv: 1207.4835] [INSPIRE]. 
[45] D.S.M. Alves, P.J. Fox and N.J. Weiner, Higgs signals in a type I 2HDM or with a sister Higgs, arXiv: 1207.5499 [INSPIRE].

[46] W. Altmannshofer, S. Gori and G.D. Kribs, A minimal flavor violating 2HDM at the LHC, Phys. Rev. D 86 (2012) 115009 [arXiv:1210.2465] [INSPIRE].

[47] S. Chang et al., Comprehensive study of two Higgs doublet model in light of the new boson with mass around $125 \mathrm{GeV}$, JHEP 05 (2013) 075 [arXiv: 1210.3439] [INSPIRE].

[48] Y. Bai, V. Barger, L.L. Everett and G. Shaughnessy, The 2HDM-X and Large Hadron Collider data, Phys. Rev. D 87 (2013) 115013 [arXiv:1210.4922] [InSPIRE].

[49] A. Drozd, B. Grzadkowski, J.F. Gunion and Y. Jiang, Two-Higgs-doublet models and enhanced rates for a $125 \mathrm{GeV}$ Higgs, JHEP 05 (2013) 072 [arXiv:1211.3580] [INSPIRE].

[50] A. Celis, V. Ilisie and A. Pich, LHC constraints on two-Higgs doublet models, JHEP 07 (2013) 053 [arXiv: 1302.4022] [INSPIRE].

[51] C.-W. Chiang and K. Yagyu, Implications of Higgs boson search data on the two-Higgs doublet models with a softly broken $Z_{2}$ symmetry, JHEP 07 (2013) 160 [arXiv:1303.0168] [INSPIRE].

[52] B. Grinstein and P. Uttayarat, Carving out parameter space in type-II two Higgs doublets model, JHEP 06 (2013) 094 [Erratum ibid. 09 (2013) 110] [arXiv:1304.0028] [INSPIRE].

[53] C.-Y. Chen, S. Dawson and M. Sher, Heavy Higgs searches and constraints on two Higgs doublet models, Phys. Rev. D 88 (2013) 015018 [arXiv:1305.1624] [INSPIRE].

[54] O. Eberhardt, U. Nierste and M. Wiebusch, Status of the two-Higgs-doublet model of type-II, arXiv: 1305.1649 [INSPIRE].

[55] N. Craig, J. Galloway and S. Thomas, Searching for signs of the second Higgs doublet, arXiv: 1305.2424 [INSPIRE].

[56] G. Bélanger, B. Dumont, U. Ellwanger, J.F. Gunion and S. Kraml, Global fit to Higgs signal strengths and couplings and implications for extended Higgs sectors, Phys. Rev. D 88 (2013) 075008 [arXiv: 1306.2941] [InSPIRE].

[57] D. López-Val, T. Plehn and M. Rauch, Measuring extended Higgs sectors as a consistent free couplings model, JHEP 10 (2013) 134 [arXiv:1308.1979] [INSPIRE].

[58] V. Barger, L.L. Everett, H.E. Logan and G. Shaughnessy, Scrutinizing the $125 \mathrm{GeV}$ Higgs boson in two Higgs doublet models at the LHC, ILC and Muon Collider, Phys. Rev. D 88 (2013) 115003 [arXiv: 1308.0052] [INSPIRE].

[59] S. Choi, S. Jung and P. Ko, Implications of LHC data on $125 \mathrm{GeV}$ Higgs-like boson for the standard model and its various extensions, JHEP 10 (2013) 225 [arXiv:1307.3948] [INSPIRE].

[60] S. Chang et al., Two Higgs doublet models for the LHC Higgs boson data at $\sqrt{s}=7$ and $8 \mathrm{TeV}$, arXiv: 1310.3374 [INSPIRE].

[61] J.R. Espinosa, C. Grojean, V. Sanz and M. Trott, NSUSY fits, JHEP 12 (2012) 077 [arXiv:1207.7355] [INSPIRE].

[62] A. Azatov, S. Chang, N. Craig and J. Galloway, Higgs fits preference for suppressed down-type couplings: implications for supersymmetry, Phys. Rev. D 86 (2012) 075033 [arXiv: 1206.1058] [INSPIRE].

[63] P. Bechtle et al., MSSM interpretations of the LHC discovery: light or heavy Higgs?, Eur. Phys. J. C 73 (2013) 2354 [arXiv:1211.1955] [INSPIRE]. 
[64] J. Cao, Z. Heng, J.M. Yang and J. Zhu, Status of low energy SUSY models confronted with the LHC $125 \mathrm{GeV}$ Higgs data, JHEP 10 (2012) 079 [arXiv: 1207.3698] [INSPIRE].

[65] H. Baer et al., Post-LHC7 fine-tuning in the minimal supergravity/CMSSM model with a 125 GeV Higgs boson, Phys. Rev. D 87 (2013) 035017 [arXiv:1210.3019] [InSPIRE].

[66] S.L. Glashow and S. Weinberg, Natural conservation laws for neutral currents, Phys. Rev. D 15 (1977) 1958 [inSPIRE].

[67] J.S. Lee et al., CPsuperH: a computational tool for Higgs phenomenology in the minimal supersymmetric standard model with explicit CP-violation, Comput. Phys. Commun. 156 (2004) 283 [hep-ph/0307377] [INSPIRE].

[68] J.S. Lee, M. Carena, J. Ellis, A. Pilaftsis and C.E.M. Wagner, CPsuperH2.0: an improved computational tool for Higgs phenomenology in the MSSM with explicit CP-violation, Comput. Phys. Commun. 180 (2009) 312 [arXiv:0712.2360] [INSPIRE].

[69] J.S. Lee, M. Carena, J. Ellis, A. Pilaftsis and C.E.M. Wagner, CPsuperH2.3: an updated tool for phenomenology in the MSSM with explicit CP-violation,

Comput. Phys. Commun. 184 (2013) 1220 [arXiv:1208.2212] [INSPIRE].

[70] R.A. Battye, G.D. Brawn and A. Pilaftsis, Vacuum topology of the two Higgs doublet model, JHEP 08 (2011) 020 [arXiv: 1106.3482] [INSPIRE].

[71] S. Davidson and H.E. Haber, Basis-independent methods for the two-Higgs-doublet model, Phys. Rev. D 72 (2005) 035004 [Erratum ibid. D 72 (2005) 099902] [hep-ph/0504050] [INSPIRE].

[72] S.Y. Choi and J.S. Lee, Decays of the MSSM Higgs bosons with explicit CP-violation, Phys. Rev. D 61 (1999) 015003 [hep-ph/9907496] [INSPIRE].

[73] S.Y. Choi, K. Hagiwara and J.S. Lee, Higgs boson decays in the minimal supersymmetric standard model with radiative Higgs sector CP-violation, Phys. Rev. D 64 (2001) 032004 [hep-ph/0103294] [INSPIRE].

[74] S.Y. Choi, M. Drees, J.S. Lee and J. Song, Supersymmetric Higgs boson decays in the MSSM with explicit CP-violation, Eur. Phys. J. C 25 (2002) 307 [hep-ph/0204200] [INSPIRE].

[75] ATLAS collaboration, Measurements of the properties of the Higgs-like boson in the four lepton decay channel with the ATLAS detector using $25 \mathrm{fb}^{1}$ of proton-proton collision data, ATLAS-CONF-2013-013 (2013).

[76] ATLAS collaboration, Search for a high-mass Higgs boson in the $H \rightarrow W W \rightarrow l \nu l \nu$ decay channel with the ATLAS detector using $21 \mathrm{fb}^{-1}$ of proton-proton collision data, ATLAS-CONF-2013-067 (2013).

[77] CMS collaboration, Search for a standard-model-like Higgs boson with a mass in the range 145 to $1000 \mathrm{GeV}$ at the LHC, Eur. Phys. J. C 73 (2013) 2469 [arXiv:1304.0213] [INSPIRE].

[78] CMS collaboration, Search for a standard model like Higgs boson in the $H \rightarrow Z Z \rightarrow 2 \ell 2 q$ decay channel at $\sqrt{s}=8 \mathrm{TeV}$, CMS-HIG-12-024 (2013).

[79] ATLAS collaboration, Search for the neutral Higgs bosons of the minimal supersymmetric standard model in pp collisions at $\sqrt{s}=7 \mathrm{TeV}$ with the ATLAS detector, JHEP 02 (2013) 095 [arXiv: 1211.6956] [INSPIRE].

[80] A. Celis, V. Ilisie and A. Pich, Towards a general analysis of LHC data within two-Higgs-doublet models, JHEP 12 (2013) 095 [arXiv:1310.7941] [inSPIRE]. 